\title{
Approximating Pareto frontier using a hybrid line search approach
}

\author{
Crina Grosan ${ }^{\mathrm{a}}$, Ajith Abraham ${ }^{\mathrm{b}, *}$

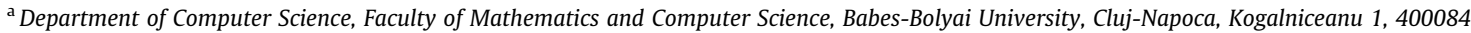 \\ Cluj-Napoca, Romania \\ ${ }^{\mathrm{b}}$ Machine Intelligence Research Labs, Scientific Network for Innovation and Research Excellence, P.O. Box 2259, Auburn, Washington, DC 98072-2259, USA
}

\section{A R T I C L E I N F O}

\section{Article history:}

Received 9 April 2009

Received in revised form 23 August 2009

Accepted 21 December 2009

\section{Keywords:}

Pareto frontier

Global optimization

Line search

Metaheuristics

Multiobjective optimization

\begin{abstract}
A B S T R A C T
The aggregation of objectives in multiple criteria programming is one of the simplest and widely used approach. But it is well known that this technique sometimes fail in different aspects for determining the Pareto frontier. This paper proposes a new approach for multicriteria optimization, which aggregates the objective functions and uses a line search method in order to locate an approximate efficient point. Once the first Pareto solution is obtained, a simplified version of the former one is used in the context of Pareto dominance to obtain a set of efficient points, which will assure a thorough distribution of solutions on the Pareto frontier. In the current form, the proposed technique is well suitable for problems having multiple objectives (it is not limited to bi-objective problems) and require the functions to be continuous twice differentiable. In order to assess the effectiveness of this approach, some experiments were performed and compared with two recent well known population-based metaheuristics namely ParEGO and NSGA II. When compared to ParEGO and NSGA II, the proposed approach not only assures a better convergence to the Pareto frontier but also illustrates a good distribution of solutions. From a computational point of view, both stages of the line search converge within a short time (average about $150 \mathrm{~ms}$ for the first stage and about $20 \mathrm{~ms}$ for the second stage). Apart from this, the proposed technique is very simple, easy to implement and use to solve multiobjective problems.
\end{abstract}

(c) 2009 Elsevier Inc. All rights reserved.

\section{Introduction}

The field of multicriteria programming abounds in methods for dealing with different kind of problems. Nevertheless, there is still space for new approaches, which can better deal with some of the difficulties encountered by the existing approaches. There are two main classes of approaches suitable for multiobjective optimization: scalarization methods and nonscalarizing methods. These approaches convert the Multiobjective Optimization Problem (MOP) into a Single Objective Optimization Problem (SOP), a sequence of SOPs, or into another MOP. There are several scalarization methods reported in the literature: weighted sum approach, weighted sth power approach, weighted quadratic approach, $\varepsilon$-constraint approach, elastic constraint approach, Benson approach, etc. are some of them [12]. Since the standard weighted sum encounters some difficulties, several other methods have been proposed to overcome the major drawbacks of this method. These include: Compromise Programming [3], Physical Programming [21-27], Normal Boundary Intersection (NBI) [4-7], and the Normal Constraint (NC) $[28,29]$ methods. There is also a huge amount of work reported on population-based metaheuristics for MOP [1,2,10-12,16,34,37-41]. Comprehensive surveys can be found in $[18,30,36]$.

\footnotetext{
* Corresponding author.

E-mail addresses: cgrosan@cs.ubbcluj.ro (C. Grosan), ajith.abraham@ieee.org (A. Abraham).
} 
In this paper, we propose a new approach, which uses a scalarization of the objectives in a way similar to the weighted sth power approach (where $s$ is 2 and the coefficients values are 1). A line search based technique is used to obtain an efficient solution. Starting with this solution, a set of efficient points are further generated, which are widely distributed along the Pareto frontier using again a line search based method but involving Pareto dominance relationship.

Empirical and graphical results and illustrations obtained by the proposed approach are compared with two well known population-based metaheuristics namely ParEGO [19] and NSGA II [8].

The paper is structured as follows: in Section 2 the proposed modified Line Search is presented. Numerical experiments considering standard benchmarks are performed in Section 3. A set of 8 multiobjective optimizations problems are considered. Sections 4 and 5 present the application of the proposed approach for solving two practical multiobjective optimization problems. Conclusions and further research plans are presented in Section 6.

\section{Line search generator of Pareto frontier}

The line search [13] is a standard and well established optimization technique [14,15]. The standard line search technique is modified in this paper so that it is able to generate the set of nondominated solutions for a MOP. The approach proposed is called Line search Generator of Pareto frontier (LGP) and it comprises of two phases: first, the problem is transformed into a SOP and a solution is found using a line search based approach. This is called as convergence phase. Second, a set of Pareto solutions are generated starting with the solution obtained at the end of convergence phase. This is called as spreading phase. The convergence and spreading phases are described below.

Consider the MOP formulated as follows:

Let $\mathfrak{R}^{m}$ and $\mathfrak{R}^{n}$ be Euclidean vector spaces referred to as the decision space and the objective space. Let $X \subset \mathfrak{R}^{m}$ be a feasible set and let $f$ be a vector-valued objective function $f: \mathfrak{R}^{m} \rightarrow \mathfrak{R}^{n}$ composed of $n$ real-valued objective functions $f=\left(f_{1}, f_{2}, \ldots, f_{n}\right)$, where $f_{k}: \mathfrak{R}^{m} \rightarrow \mathfrak{R}$, for $k=1,2, \ldots, n$. A MOP is given by:

$\min \left(f_{1}(x), f_{2}(x), \ldots, f_{n}(x)\right)$

subject to $x \in X$.

\subsection{Convergence phase}

The MOP is transformed into a SOP by aggregating the objectives using an approach similar to the weighted $t$ th power approach. We consider $s=2$ and the values of weights equal to 1 . The obtained SOP is:

$\min \quad F=\sum_{i=1}^{n} f_{i}^{2}(x)$

subject to $x \in X$.

Remark. In our experiments the value 2 for $s$ fits very well. But when negative objectives are to be taken into consideration, then an odd value should be used for $s$ (an even value can alter the results).

A modified line search method is used to find the optimum of this problem. The modification proposed in this paper for the standard line search technique refers to direction and step setting and also the incorporation of a re-start procedure. To fine tune the performance, the first partial derivatives of the function to optimize are also made use of. The proposed modifications refer to:

- The setting of the direction and step.

- The re-starting of the line search method.

After a given number of iterations, the process is re-started by reconsidering other arbitrary starting point which is generated by taking into account the result obtained at the end of previous set of iterations.

\subsubsection{Direction and step setting}

Initially, several experiments were performed in order to set an adequate value for the direction. The standard value +1 or -1 was used and for some functions the value -1 was favourable to obtain good performance. Some experiments were also performed by setting the direction value as being a random number between 0 and 1 . It was found that the usage of random number helped to obtain overall very good performance for the entire considered test functions. But usage of the value -1 for direction, obtains almost the same performance similar to that obtained with a random value. So, either of these values (the random one and the value -1 ) may be used for better performance.

The step is set as follows:

$$
\alpha_{k}=2+\frac{3}{2^{2 k}+1},
$$


where $k$ refers to the iteration number.

The modified line search technique is summarized as follows:

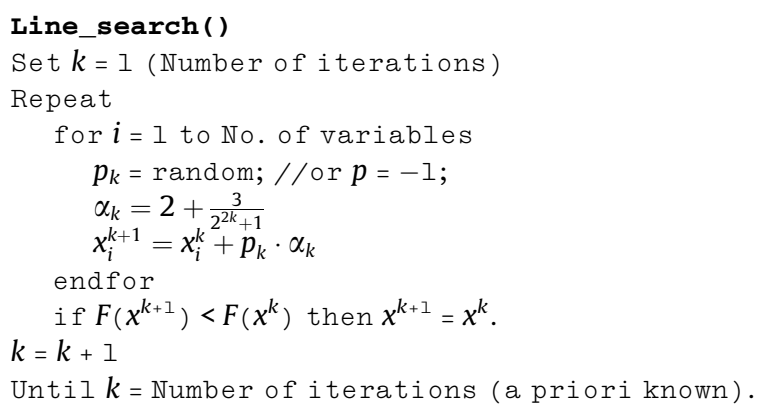

\section{Remarks}

(i) The condition:

if $F\left(x^{k+1}\right)<F\left(x^{k}\right)$ then $x^{k+1}=x^{k}$

allows to move to the new generated point only if there is an improvement in the quality of the objective function.

(ii) Number of iterations for which line search is applied is apriori known and is usually a small number. For the experiments reported in this paper, the number of these iterations was set to 10.

(iii) When re-starting the line search method (after the insertion of the re-start technique) the value of the iterations number starts again from 1 (this should not be related to the value of $\alpha$ after the first set of iterations (and after each of the following iterations)).

Several experiments were attempted to set a value for the step, starting with random values (until a point is reached for which the objective function achieves a better value); using a starting value for the step and generating random numbers with Gaussian distribution around this number, etc. As a result of the initial experiments performed, it was decided to use Eq. (1) to compute the step size. But, of course, there are also several other ways to set this.

\subsubsection{Incorporation of re-start procedure}

In order to re-start the algorithm the result obtained in the previous set of iterations (denote it by $x$ ) is taken into account and the steps given below are followed:

1. For each dimension $i$ of the point $x$, the first partial derivative with respect to this dimension is calculated. This means the gradient of the objective function is calculated which is denoted by $g$. Taking this into account, the bounds of the definition domain for each dimension are re-calculated as follows:

$$
\begin{aligned}
& \text { if } g_{i}=\frac{\partial F}{\partial x_{i}}>0 \text { then } \text { upper bound }=x_{i} ; \\
& \text { if } g_{i}=\frac{\partial F}{\partial x_{i}}<0 \text { then lower bound }=x_{i} .
\end{aligned}
$$

2. The search process is re-started by re-initializing a new arbitrary point between the newly obtained boundaries.

\subsection{Spreading phase}

At the end of the convergence phase, a solution is obtained. This solution is considered as an efficient (or Pareto) solution. During this phase and taking into account of the existing solution, more efficient solutions are to be generated so as to have a thorough distribution of all several good solutions along the Pareto frontier. In this respect, the line search technique is made use of to generate one solution at the end of each set of iterations. This procedure is applied several times in order to obtain a larger set of nondominated solutions. The following steps are repeated in order to obtain one nondominated solution:

Step 1. A set of nondominated solutions found so far is archived. Let us denote it by NonS. Initially, this set will have the size one and will only contain the solution obtained at the end of convergence phase.

Step 2. We apply line search for one solution and one dimension of this solution at one time. For this:

Step 2.1. A random number $i$ between one and $|\operatorname{NonS}|(|\cdot|$ denotes the cardinal $)$ is generated. Denote the corresponding solution by nons . $_{\text {. }}$ 


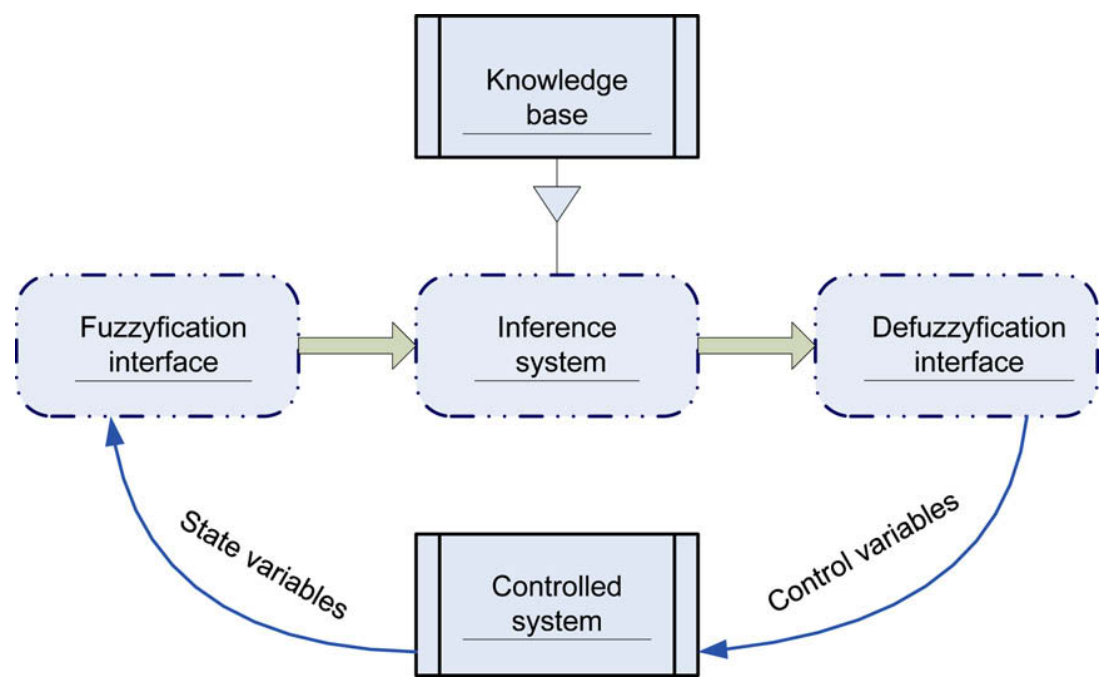

Fig. 1. Generic structure of an FLC.

Step 2.2. A random number $j$ between one and the number of dimensions (the number of decision variables) is generated. Denote this by nons $i j$.

Step 3. Line search is applied for nons $i j$.

Step 3.1. Set a random value for $p$ between $[-0.5,1]$.

Step 3.2. Set $\alpha$ (which depends on the problem, on the number of total nondominated solutions which are to be generated, etc.).

Step 3.3. The new obtained solution new_sol is identical to $n o n S_{i}$ in all dimensions except dimension $j$ which is:

$$
\text { new } \_ \text {sol } l_{j}=n o n S_{i j}+\alpha \cdot p \text {. }
$$

Step 3.4. If (new_sol $l_{j}>$ upper bound) or (new_sol $l_{j}<$ lower bound)then new_sol $l_{j}=l o w e r$ bound + random $\cdot($ upper bound - lower bound).

Step 4. If $F\left(n e w \_s o l\right)>F\left(n o n S_{1}\right)$ then discard new_solelse if new_sol is nondominated with respect to the set NonS then add new_sol to NonS and increase the size on NonS by 1 .

Go to step 2.

Step 5. Stop

These steps are repeated until a set on nondominated solutions of a required size is obtained. In our experiments the size of this set is 100 .

Note that this procedure it very fast and it takes less than $20 \mathrm{~ms}$ to obtain 100 nondominated solutions.

\subsubsection{Estimating the value of $\alpha$ using a fuzzy logic controller}

The use of fuzzy logic controllers to adapt the $\alpha$ value is useful to improve the performance. An FLC is composed by a knowledge base, that includes the information given by the expert in the form of linguistic control rules, a fuzzyfication interface, which has the effect of transforming crisp data into fuzzy sets, an inference system, that uses them together with the knowledge base to make inference by means of a reasoning method, and a defuzzyfication interface, that translates the fuzzy control action thus obtained to a real control action using a defuzzyfication method. The generic structure of an FLC is shown in Fig. 1.

In order to set an adequate value for $\alpha$ so that the solutions will have a good distribution on the Pareto front, we are proceeding as follows:

- Select a sample set of solutions uniform distributed on the Pareto front (denoted by SPS) of size equal to the size of the approximation set obtained by the our approach.

- For each point from the approximation set obtained by our approach identify the closest point in SPS.

- Mark each such identified point from SPS.

- Set the value of distribution indices $\left(D_{i}\right)$ as being equal to the number of marked points from SPS.

Our strategy for updating the $\alpha$ value is to consider the changes of the value of maximum distribution indices $\left(D_{i m}\right)$ and average distribution indices $\left(D_{i a}\right)$ in two consecutive iterations. The performance may be measured using two error indices: 

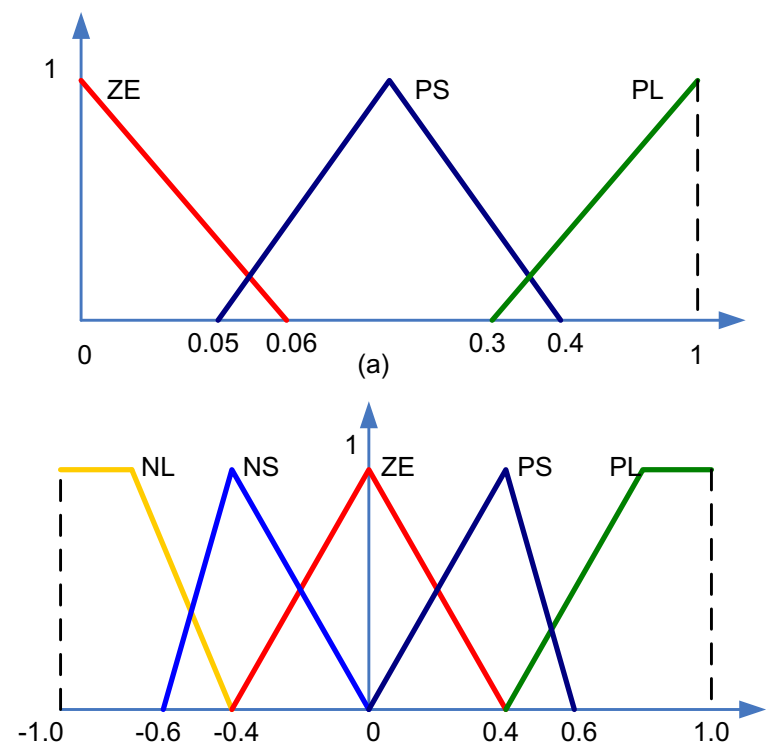

(b)

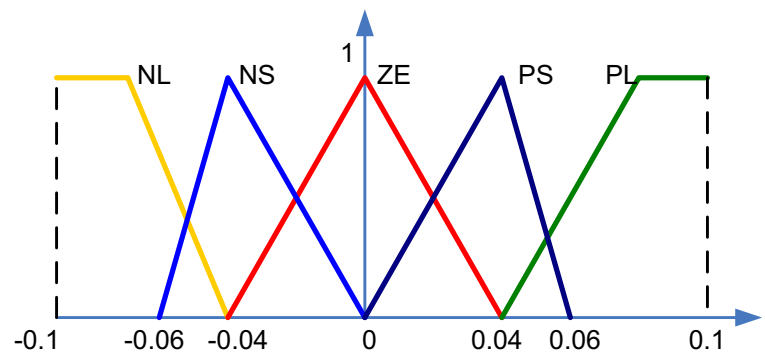

(c)

Fig. 2. Membership functions. (a) For $e_{1}$; (b) $e_{2}$; (c) for $\Delta \alpha$.

$$
\begin{aligned}
& e_{1}(t)=\frac{D_{i m}(t)-D_{i a}(t)}{D_{i m}(t)}, \\
& e_{2}(t)=\frac{D_{i a}(t)-D_{i a}(t-1)}{D_{i m}(t)},
\end{aligned}
$$

where $t$ is time step, $D_{i m}(t)$ is the maximum distribution index at iteration $t, D_{i a}(t)$ is the average distribution index at iteration $t$ and $D_{i a}(t-1)$ is the average distribution index at iteration $(t-1)$.

A two-dimension FLC system is used, in which there are two parameters $e_{1}$ and $e_{2}$. The membership functions are shown in Fig. 2, where $N L$ is Negative large, $N S$ is Negative small, ZE is Zero, PS is Positive small and PL is Positive large.

For the controlling the performance, the output $\alpha(t)$ of the fuzzy logic controller is translated using fuzzy if-then rules as illustrated in Fig. 3. Center of gravity is used as defuzzyfication method. Then we use the crisp value to modify the parameters $\alpha$ as follows:

$$
\alpha(t)=\alpha(t-1)+\Delta \alpha
$$

For applying the procedure described above, the Pareto front it is supposed to be known (and this is the case in all our experiments considered). In Fig. 4, two approximation sets $A$ and $B$ and a sample set of Pareto points (SPS) of size 10 are considered. The value of $D_{i}$ for the set $A$ is 6 (which means 6 solutions from the SPS are marked) while the value of $D_{i}$ for the set $B$ is 10. This means set $\mathrm{B}$ is obtaining a better distribution on the Pareto front than the set $A$.

Example. An illustrative example for calculation of $\alpha$ is presented in Fig. 5. Suppose we have an approximation set containing 100 solutions. This involves the size of the selected Pareto sample points will be also 100. Let us also suppose that are at the third iteration and we obtained the value 60 for $D_{i}$ for $\alpha=0.1$ in the first iteration and value 63 for $D_{i}$ for $\alpha=0.15$ in the second iteration. It should be mentioned that the value of $\alpha$ in the first iteration was randomly chosen and for the value of $\alpha$ in the second iteration we had the chance to increase or decrease de current value; so, we increased it to 0.15 . 


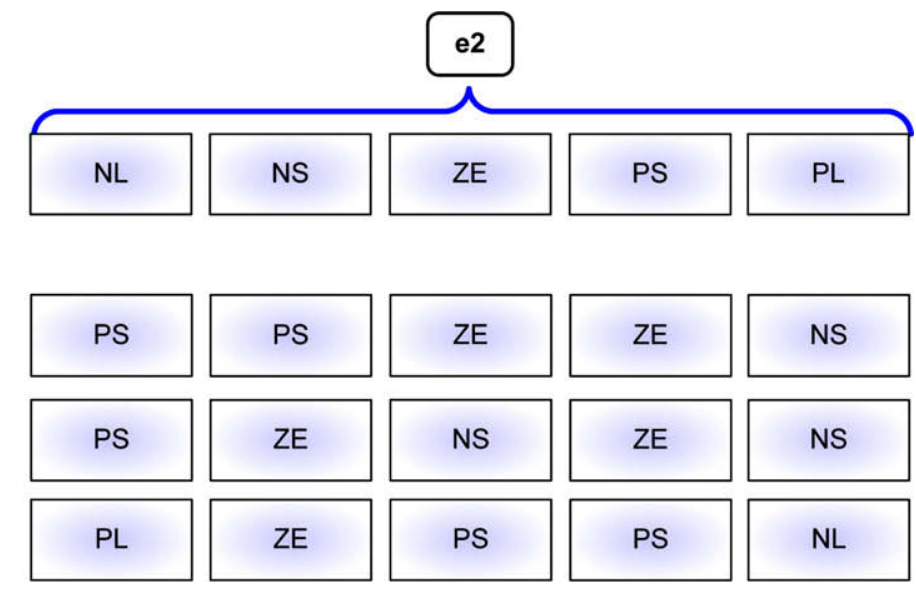

Fig. 3. Fuzzy rules for $\Delta \alpha$.
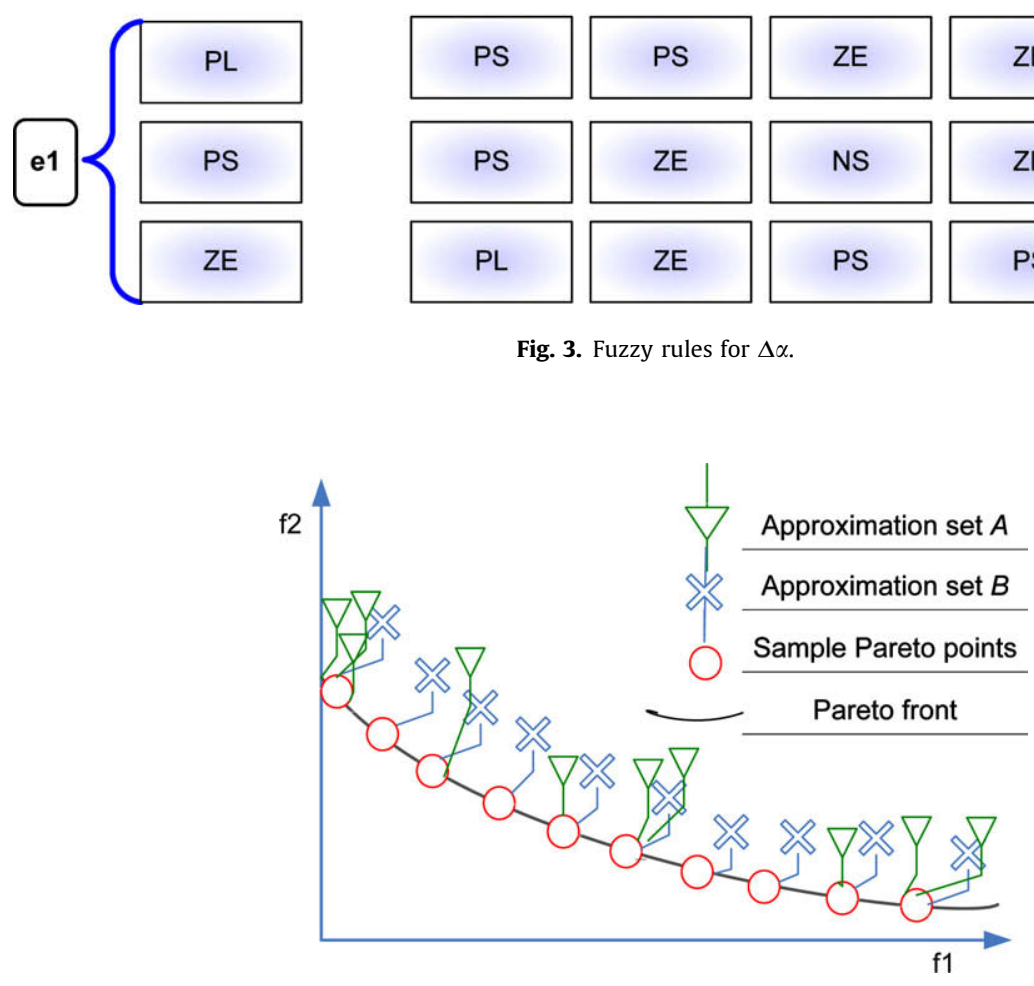

Fig. 4. Illustration of Pareto approximation used for $D_{i}$ calculation.

Starting with the third iteration we can apply our fuzzy rules to calculate the next value for $\alpha$. By using the formulas (2) and (3), the membership functions from Fig. 2 and the fuzzy rules from Fig. 3, the value 0.04 for $\Delta \alpha$ is obtained. Based on this, the value of $\alpha$ at the iteration 3 will be $0.15+0.04=0.19$.

\section{Experiments and comparisons}

In order to assess the performance of LGP, some experiments were performed using some well known bi-objective and three-objective test functions [17,31], which are adapted from $[9,16]$. These test functions were also used by the authors of ParEGO [19] and NSGA II [8], which are well known in the computational intelligence community as very efficient techniques for multiobjective optimization. Details about implementation of these two techniques may obtained from [8,19]. Parameters used by ParEGO and NSGA II (given in Table 1) and the results obtained by these two techniques are adapted from [19].

A set of 100 nondominated solutions obtained by LGP, ParEGO, NSGA II is compared in terms of dominance and convergence to the Pareto set. For the first comparison, two indices were computed for each set of two comparisons: number of solution obtained by the first technique which dominate solutions obtained by the second technique and number of solutions obtained by the first technique which are dominated by the solutions obtained by the second technique.

For two sets of $A$ and $B$ of solutions, which are compared, indices are denoted by $\operatorname{Dominate}(A, B)$ and $\operatorname{Dominated}(A, B)$, respectively. Visualization plots are used to illustrate the distribution of solutions on the Pareto frontier.

LGP uses only three parameters:

- number of re-starts: 20 (10 for KNO1);

- number of iteration per each re-start: 10 ;

- $\alpha$ for the spreading phase (which is set independent for each test function). 


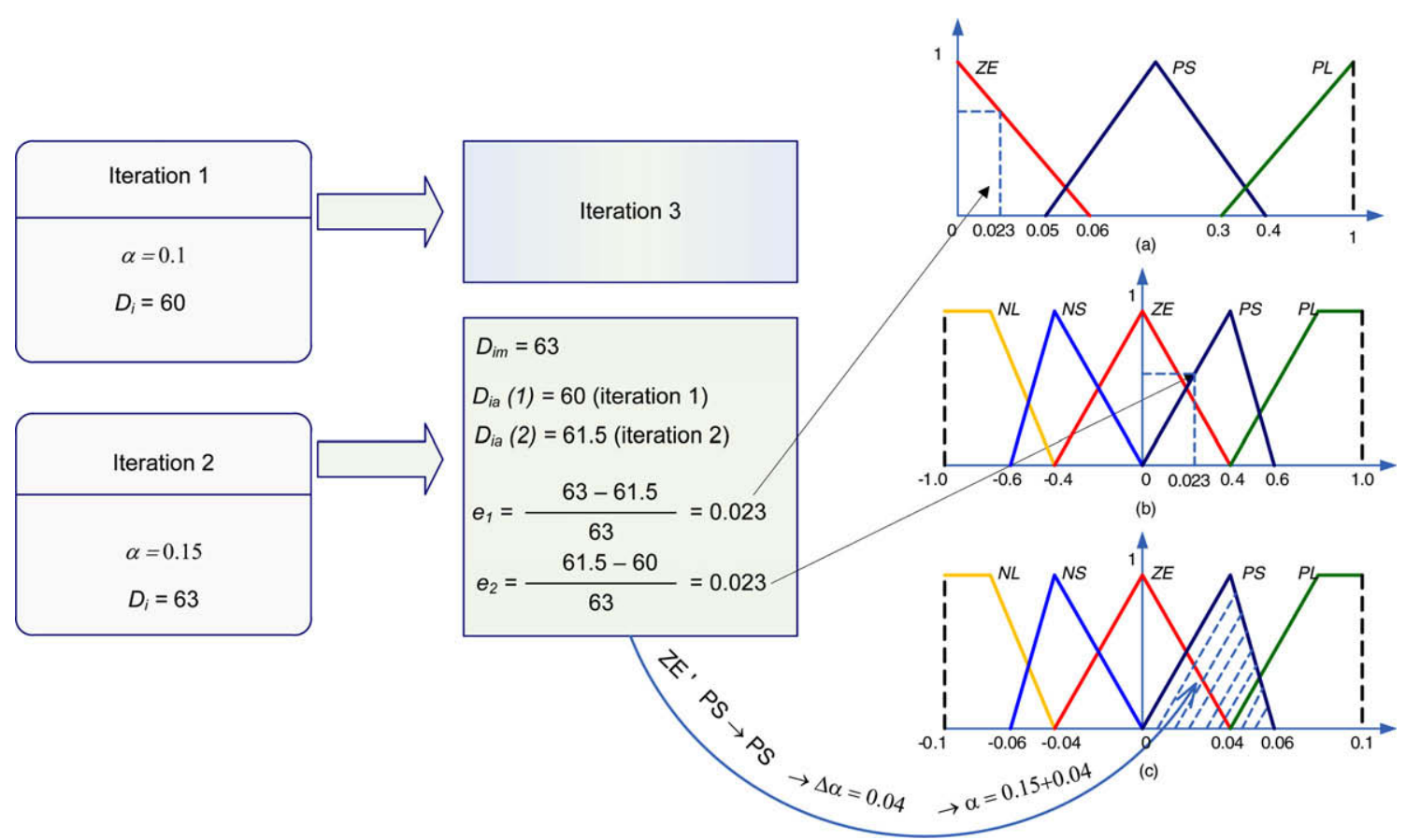

Fig. 5. Example of $\alpha$ calculation.

Table 1

Parameters used in experiments by ParEGO and NSGA II. $d$ denotes the number of decision parameter dimensions.

\begin{tabular}{|c|c|c|c|}
\hline \multicolumn{2}{|l|}{ ParEGO } & \multicolumn{2}{|l|}{ NSGA II } \\
\hline Parameter & Value & Parameter & Value \\
\hline Initial population in latin hypercube & $11 d-1$ & Population size & 20 \\
\hline Total maximum evaluations & 250 & Maximum generations & 13 \\
\hline Number of scalarizing vectors & $\begin{array}{l}11 \text { for } 2 \text { objectives } \\
15 \text { for } 3 \text { objectives }\end{array}$ & Crossover probability & 0.9 \\
\hline Scalarizing function & Augmented Tchebycheff & Real value mutation probability & $1 / d$ \\
\hline Internal genetic algorithm evaluations per iteration & 200,000 & Real value SBX parameter & 10 \\
\hline Crossover probability & 0.2 & Real value mutation parameter & 50 \\
\hline Real value mutation probability & $1 / d$ & & \\
\hline Real value SBX parameter & 10 & & \\
\hline Real value mutation parameter & 50 & & \\
\hline
\end{tabular}

\subsection{Test function KNO1}

This test function has two variables and two objectives. It is given by:

$$
\begin{aligned}
& \text { minimize } f_{1}=20-r \cdot \cos (\phi), \\
& \text { minimize } f_{2}=20-r \cdot \sin (\phi),
\end{aligned}
$$

where

$$
\begin{aligned}
& r=9-\left(3 \sin \left(\frac{5\left(x_{1}+x_{2}\right)^{2}}{2}\right)+3 \sin \left(4\left(x_{1}+x_{2}\right)\right)+5 \sin \left(2\left(x_{1}+x_{2}\right)\right)+2\right), \\
& \phi=\frac{\pi\left(x_{1}-x_{2}+3\right)}{12} .
\end{aligned}
$$

The distance from the Pareto front is controlled by $r$ and is a function of the sum of the decision variables. The location transverse to the Pareto front is controlled by the difference between the decision variables. Pareto set consists of all pairs whose sum is 4.4116 . There are 15 local Pareto fronts and the true Pareto front lies just beyond a local Pareto front which has a larger basin of attraction. 

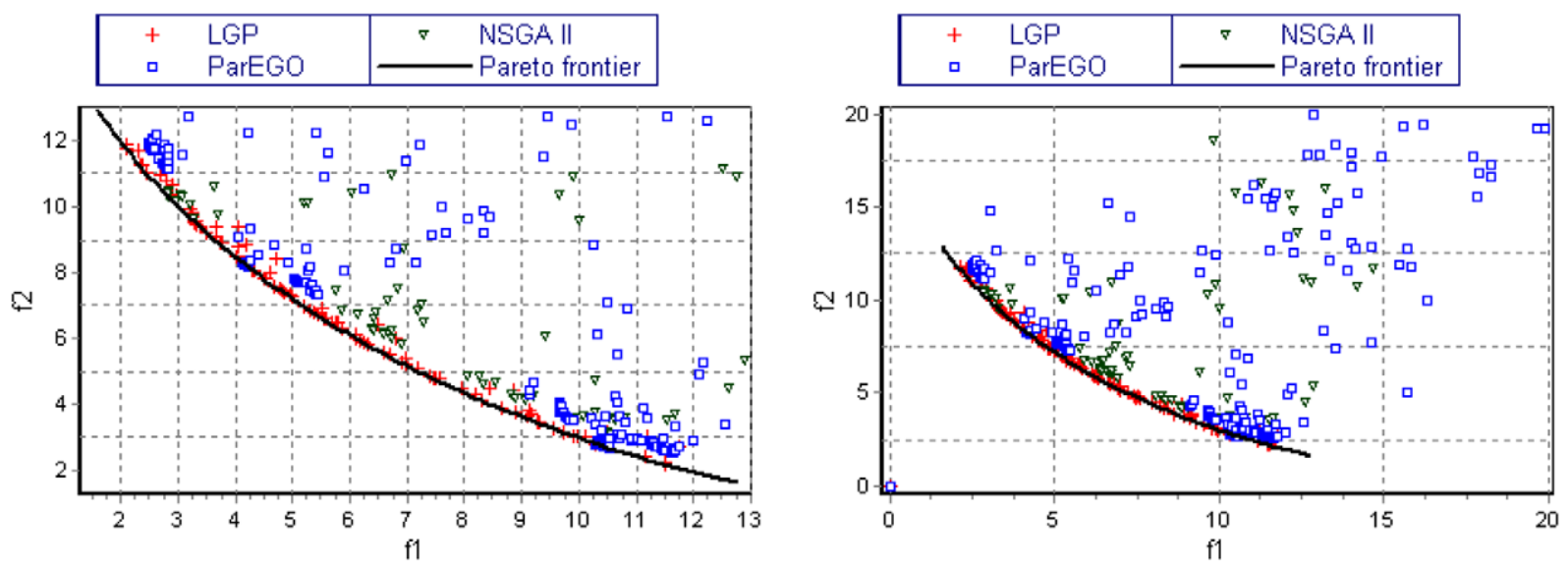

Fig. 6. Distribution of solutions on the Pareto frontier obtained by LGP, ParEGO and NSGA II for test function KNO1.

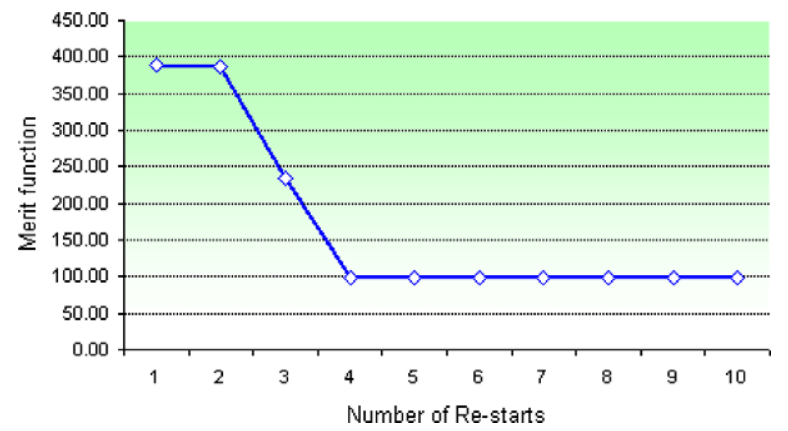

Fig. 7. Behavior of the merit function for test function KNO1 during the convergence phase.

Table 2

The dominance between solutions obtained by LGP, ParEGO and NSGA II for test function KNO1.

\begin{tabular}{|c|c|c|c|c|c|c|c|c|}
\hline Dominate & ParEGO & NSGA II & Dominate & LGP & NSGA II & Dominate & LGP & ParEGO \\
\hline LGP & 100 & 100 & ParEGO & 7 & 59 & NSGA II & 2 & 42 \\
\hline Dominated & ParEGO & NSGA II & Dominated & LGP & NSGA II & Dominated & LGP & ParEGO \\
\hline LGP & 7 & 2 & ParEGO & 100 & 42 & NSGA II & 100 & 59 \\
\hline
\end{tabular}

The convergence to the Pareto frontier and the distribution of solutions obtained by LGP, ParEGO and NSGA II for the test function DTLZ1a is depicted in Fig. 6. Different sizes of the objective space are illustrated in order to incorporate all solutions obtained by all techniques. The value 0.6 is considered for $\alpha$ for this test function. The behavior of the merit function during the 10 re-starts is depicted in Fig. 7. From the results presented in Table 2 it can be observed that 7 of the solutions obtained by LGP are dominated by solutions obtained by ParEGO and 2 are dominated by solutions obtained by NSGA II. Solutions obtained by LGP dominate all 100 solutions obtained by both ParEGO and NSGA II. 59 of the solutions obtained by NSGA II are dominated by solutions obtained by ParEGO while 42 of the solutions obtained by ParEGO are dominated by solutions obtained by NSGA II.

\subsection{Test function $O K A 1$}

This test function and the test function OKA2 have been proposed in [29]. It is a bi-objective test function having two variables and it is defined as:

$$
\begin{aligned}
& \text { minimize } f_{1}=x_{1}^{\prime}, \\
& \text { minimize } f_{2}=\sqrt{2 \pi}-\sqrt{\left|x_{1}^{\prime}\right|}+2\left|x_{2}^{\prime}-3 \cos \left(x_{1}^{\prime}\right)-3\right|^{\frac{1}{3}},
\end{aligned}
$$



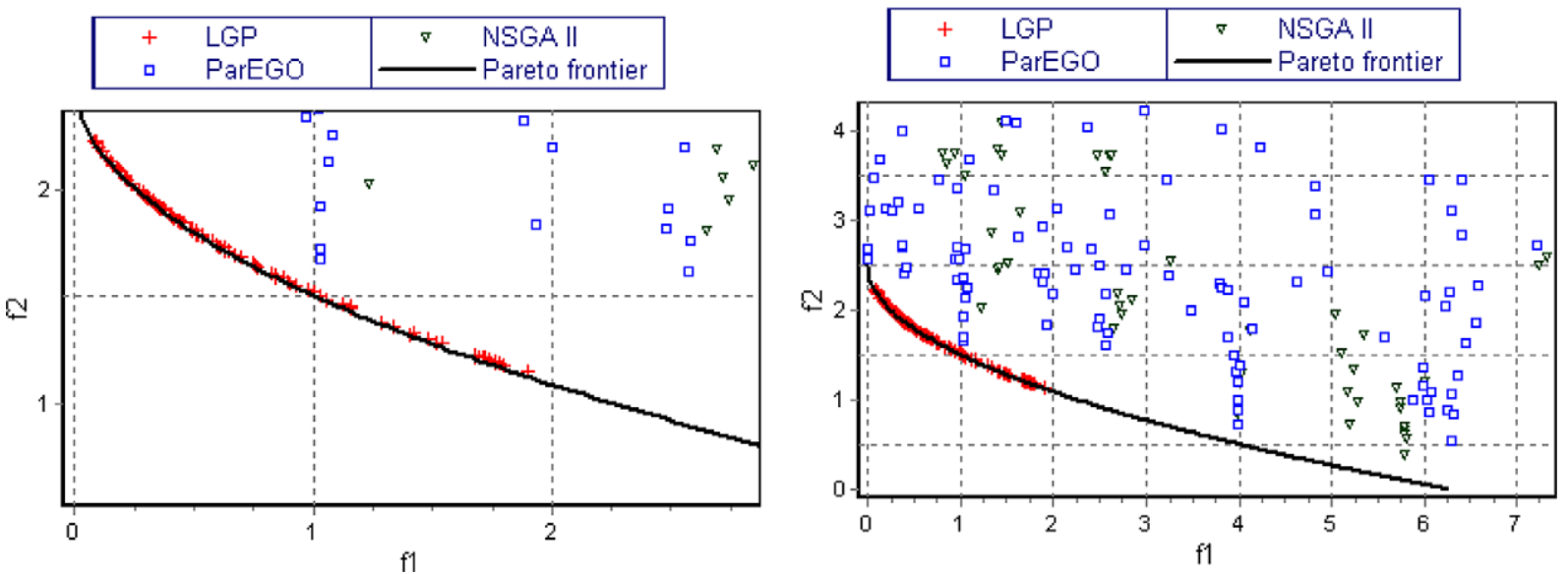

Fig. 8. Distribution of solutions on the Pareto frontier obtained by LGP, ParEGO and NSGA II for test function OKA1.

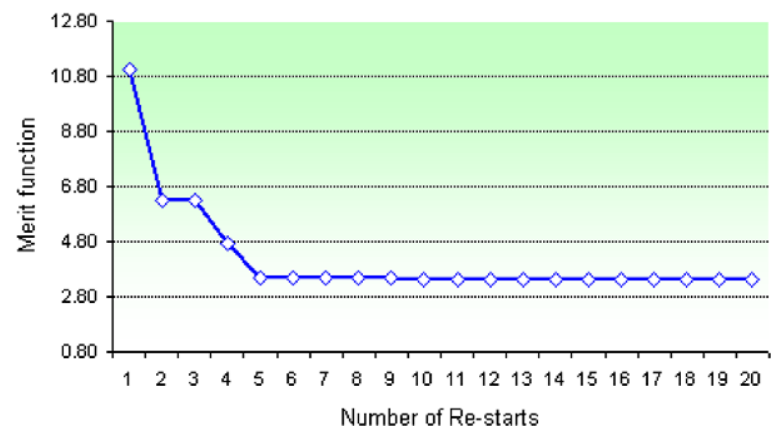

Fig. 9. Behavior of merit function for test function OKA1 during the convergence phase.

Table 3

The dominance between solutions obtained by LGP, ParEGO and NSGA II for test function OKA1.

\begin{tabular}{lllllllll}
\hline Dominate & ParEGO & NSGA II & Dominate & LGP & NSGA II & Dominate & LGP & ParEGO \\
LGP & 83 & 64 & ParEGO & 0 & 77 & NSGA II & 0 & 59 \\
Dominated & ParEGO & NSGA II & Dominated & LGP & NSGA II & Dominated & LGP & ParEGO \\
LGP & 0 & 0 & ParEGO & 83 & 59 & NSGA II & 64 & 77 \\
\hline
\end{tabular}

where

$$
\begin{aligned}
& x_{1}^{\prime}=\cos \left(\frac{\pi}{12}\right) x_{1}-\sin \left(\frac{\pi}{12}\right) x_{2} \\
& x_{2}^{\prime}=\sin \left(\frac{\pi}{12}\right) x_{1}+\cos \left(\frac{\pi}{12}\right) x_{2}, \\
& x_{1} \in\left[6 \sin \left(\frac{\pi}{12}\right), 6 \sin \left(\frac{\pi}{12}\right)+2 \pi \cdot \cos \left(\frac{\pi}{12}\right)\right], \\
& x_{2} \in\left[-2 \pi \cdot \sin \left(\frac{\pi}{12}\right), 6 \cos \left(\frac{\pi}{12}\right)\right] .
\end{aligned}
$$

The Pareto optimal set lies on the curve $x_{2}^{\prime}=3 \cos \left(x_{1}^{\prime}\right)+2, x_{1}^{\prime} \in[0,2 \pi]$.

The solutions obtained by LGP, ParEGO and NSGA II for the test function DTLZ1a are depicted in Fig. 8. Different sizes of the objective space are illustrated in order to incorporate all solutions obtained by all techniques. The value of parameter $\alpha$ used by LGP in the spreading phase is set to 1 . The behavior of the merit function during the 20 re-starts is depicted in Fig. 9. From the results presented in Table 3 it can be observed that none of the solutions obtained by LGP are dominated by solutions obtained by either ParEGO or NSGA II. Solutions obtained by LGP dominate 83 solutions obtained by both ParEGO and 64 solutions obtained by NSGA II. Seventy-seven of the solutions obtained by NSGA II are dominated by solutions obtained by ParEGO while 59 of the solutions obtained by ParEGO are dominated by solutions obtained by NSGA II. 


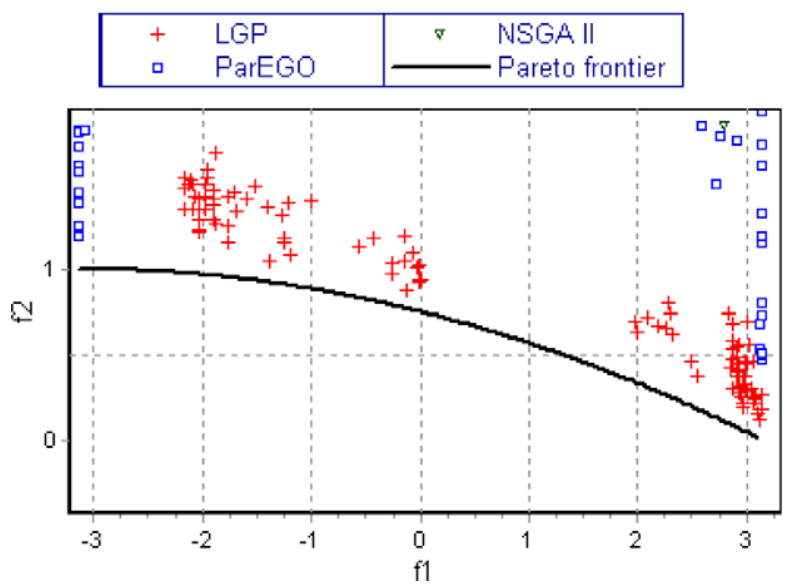

\begin{tabular}{|cl|cc|}
\hline+ & LGP & & NSGA II \\
a & ParEGO & & \\
\cline { 2 - 4 }
\end{tabular}

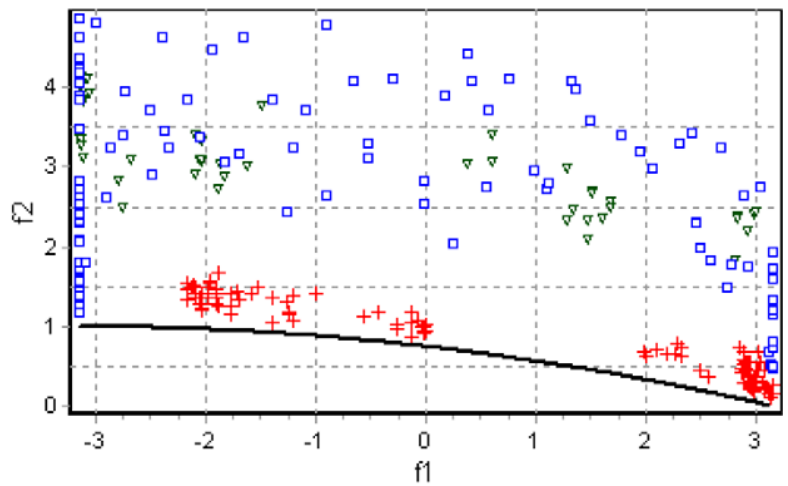

Fig. 10. Distribution of solutions on the Pareto frontier obtained by LGP, ParEGO and NSGA II for test function OKA2.

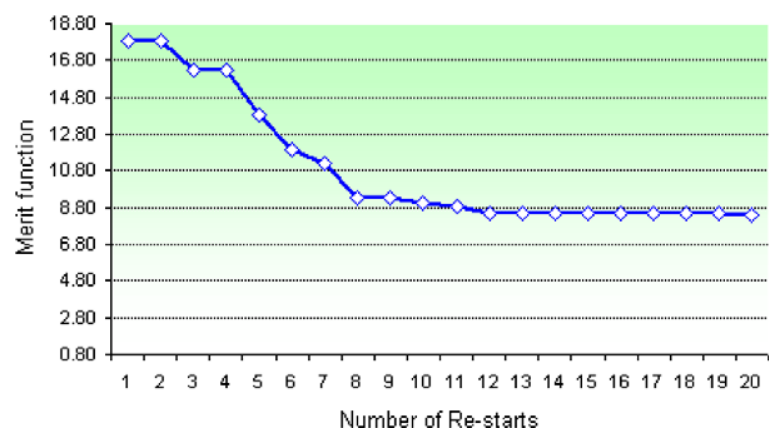

Fig. 11. Behavior of merit function for test function OKA2 during the convergence phase.

Table 4

The dominance between solutions obtained by LGP, ParEGO and NSGA II for test function OKA2.

\begin{tabular}{llllllll}
\hline Dominate & ParEGO & NSGA II & Dominate & LGP & NSGA II & Dominate \\
LGP & 37 & 41 & ParEGO & 28 & 31 & LGP & NSGA II \\
Dominated & ParEGO & NSGA II & Dominated & LGP & NSGA II & Dominated \\
LGP & 28 & 0 & ParEGO & 37 & 69 & LGP & NSGA II \\
\hline
\end{tabular}

\subsection{Test function $\mathrm{OKA2}$}

Test function OKA2 is given by:

$$
\begin{aligned}
& \text { minimize } f_{1}=x_{1}, \\
& \text { minimize } f_{2}=1-\frac{1}{4 \pi^{2}}\left(x_{1}+\pi\right)^{2}+\left|x_{2}-5 \cdot \cos \left(x_{1}\right)\right|^{\frac{1}{3}}+\left|x_{3}-5 \cdot \sin \left(x_{1}\right)\right|^{\frac{1}{3}}, \\
& x_{1} \in[-\pi, \pi], \quad x_{2}, x_{3} \in[-5,5] .
\end{aligned}
$$

The Pareto optima lie on a spiral-shaped curve in the three-dimensional variable space.

The Pareto front is given by

$$
f_{2}=1-\frac{1}{4 \pi^{2}}\left(f_{1}+\pi\right)^{2}, \quad f_{1} \in[-\pi, \pi] .
$$

The convergence to the Pareto frontier and the distribution of solutions obtained by LGP, ParEGO and NSGA II for the test function DTLZ1a is depicted in Fig. 10. The value 1 is considered for $\alpha$ for this test function. The behavior of the merit function during the 20 re-starts is depicted in Fig. 11. From the results presented in Table 4 it can be observed that 28 of the solutions obtained by LGP are dominated by solutions obtained by ParEGO while 37 solutions obtained by ParEGO and 41 solutions 

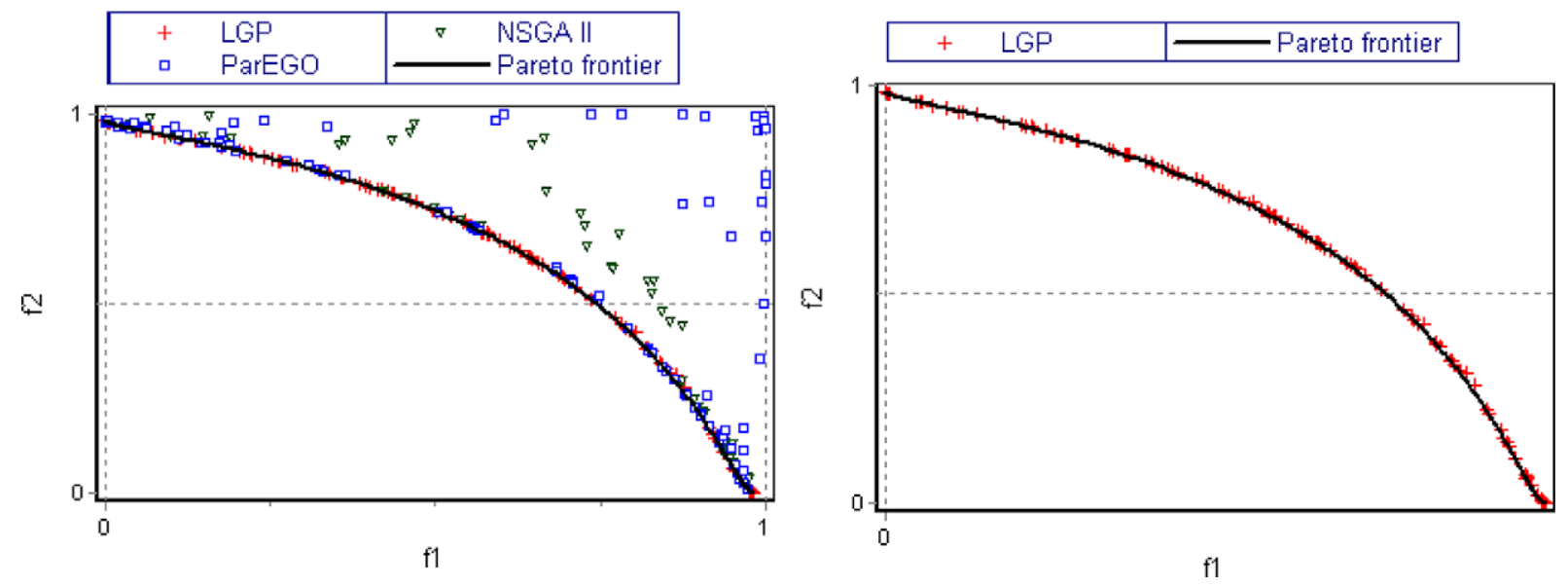

Fig. 12. Distribution of solutions on the Pareto frontier obtained by LGP, ParEGO and NSGA II for test function VLMOP2.

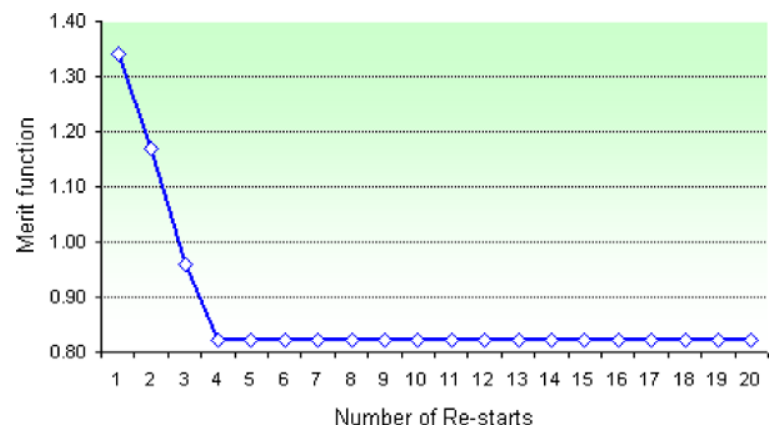

Fig. 13. Behavior of merit function for test function VLMOP2 during the convergence phase.

obtained by NSGA II are dominated by solutions obtained by LGP. Thirty-one of the solutions obtained by NSGA II are dominated by solutions obtained by ParEGO while 69 of the solutions obtained by ParEGO are dominated by solutions obtained by NSGA II.

It can be observed that this function is a difficult one. LGP converges very close to the Pareto frontier but does not obtain a very good distribution of solutions while both ParEGO and NSGA II are far from the frontier.

\subsection{Test function VLMOP2}

This test function has been proposed in [39]. It is a bi-objective problem having scalable number decision variables. We use two variables:

$$
\begin{aligned}
& \text { minimize } f_{1}=1-\exp \left(\sum_{i=1}^{n}\left(x_{i}-\frac{1}{\sqrt{n}}\right)^{2}\right), \\
& \text { minimize } f_{2}=1-\exp \left(\sum_{i=1}^{n}\left(x_{i}+\frac{1}{\sqrt{n}}\right)^{2}\right), \\
& x_{1}, x_{2} \in[-2,2], \quad n=2 .
\end{aligned}
$$

The Pareto front is concave and the Pareto optima lie on the diagonal passing from $\left(-\frac{1}{\sqrt{n}},-\frac{1}{\sqrt{n}}\right)$ to $\left(\frac{1}{\sqrt{n}}, \frac{1}{\sqrt{n}}\right)$ in the decision variable space.

The convergence to the Pareto frontier and the distribution of solutions obtained by LGP, ParEGO and NSGA II for the test function DTLZ1a is depicted in Fig. 12. The value 1 is considered for $\alpha$ for this test function. The behavior of the merit function during the 20 re-starts is depicted in Fig. 13. From the results presented in Table 5 it can be observed that six of the solutions obtained by LGP are dominated by solutions obtained by ParEGO. Solutions obtained by LGP dominate 49 solutions obtained by ParEGO and 75 solutions obtained by NSGA II. Seventy-five of the solutions obtained by NSGA II are dominated by solutions obtained by ParEGO while 37 of the solutions obtained by ParEGO are dominated by solutions obtained by NSGA II. 
Table 5

The dominance between solutions obtained by LGP, ParEGO and NSGA II for test function VLMOP2.

\begin{tabular}{llllllll}
\hline $\begin{array}{llll}\text { Dominate } \\
\text { LGP }\end{array}$ & ParEGO & NSGA II & Dominate & LGP & NSGA II & Dominate & LGP \\
Dominated & 49 & 75 & ParEGO & 6 & 75 & NSGA II \\
LGP & ParEGO & NSGA II & Dominated & LGP & NSGA II & Dominated & LGP \\
\hline
\end{tabular}
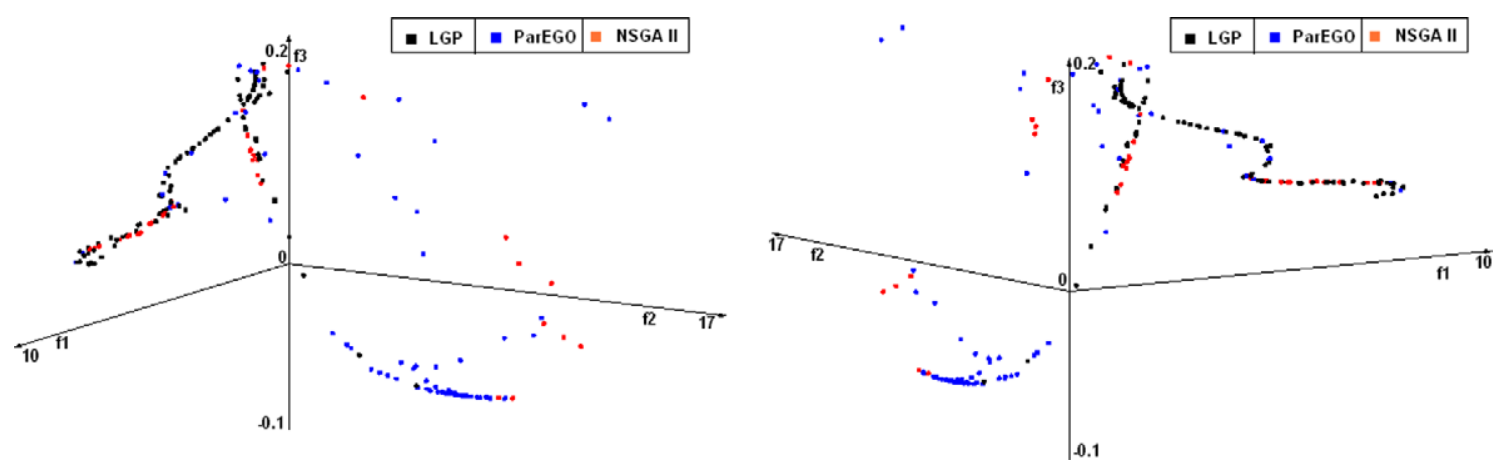

Fig. 14. Pareto front obtained by LGP, ParEGO and NSGA II for the test function VLMOP3.

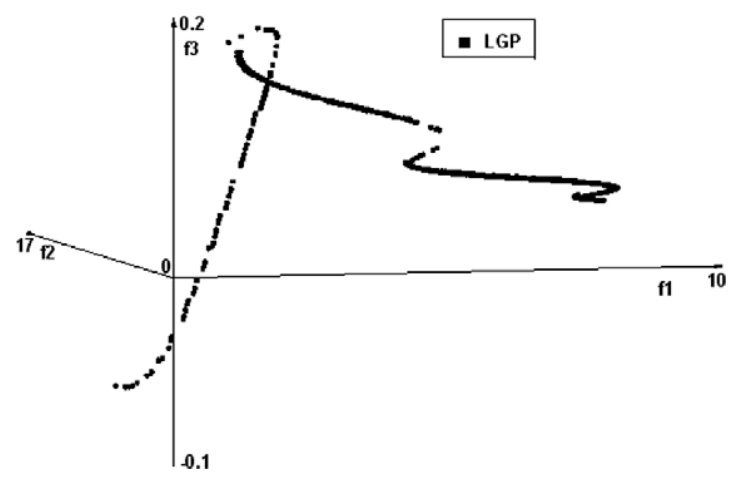

Fig. 15. Pareto front obtained by LGP for VLMOP3 test problem with a final set of 1000 solutions.

\subsection{Test function VLMOP3}

Test function VLMOP3 has been proposed by Veldhuizen and Lamont [39] and consists of three-objective functions of two variables given by:

$$
\begin{aligned}
& \text { minimize } f_{1}=0.5\left(x^{2}+y^{2}\right)+\sin \left(x^{2}+y^{2}\right) \\
& \text { minimize } f_{2}=\frac{(3 x-2 y+4)^{2}}{8}+\frac{(x-y+1)^{2}}{27}+15 \\
& \text { minimize } f_{2}=\frac{1}{x^{2}+y^{2}+1}-1.1 \cdot \exp \left(-x^{2}-y^{2}\right) \\
& x, y \in[-3,3]
\end{aligned}
$$

This test function has a disconnected Pareto optimal set and the Pareto-optimal front is a curve following a convoluted path through objective space.

The convergence to the Pareto frontier and the distribution of solutions obtained by LGP, ParEGO and NSGA II for the test function DTLZ1a is depicted in Fig. 14. The value of $\alpha$ for this test function is 1. Pareto front obtained by LGP considering a set of 1000 solutions is depicted in Fig. 15. The behavior of the merit function during the 20 re-starts is depicted in Fig. 16. From the results presented in Table 6 it can be observed that six of the solutions obtained by LGP are dominated by solutions obtained by ParEGO. Solutions obtained by LGP dominate 49 solutions obtained by ParEGO and 75 solutions obtained by NSGA II. Seventy-five of the solutions obtained by NSGA II are dominated by solutions obtained by ParEGO while 37 of the solutions obtained by ParEGO are dominated by solutions obtained by NSGA II. 


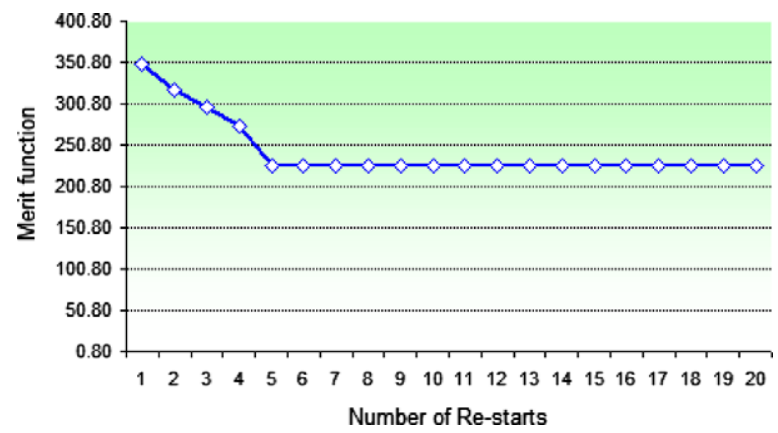

Fig. 16. Behavior of merit function for test function VLMOP3 during the convergence phase.

Table 6

The dominance between solutions obtained by LGP, ParEGO and NSGA II for test function VLMOP3.

\begin{tabular}{lllllllll}
\hline Dominate & ParEGO & NSGA II & Dominate & LGP & NSGA II & Dominate & LGP & ParEGO \\
LGP & 44 & 91 & ParEGO & 15 & 33 & NSGA II & 20 & 39 \\
Dominated & ParEGO & NSGA II & Dominated & LGP & NSGA II & Dominated & LGP & ParEGO \\
LGP & 15 & 20 & ParEGO & 44 & 39 & NSGA II & 91 \\
\hline
\end{tabular}

\subsection{Test function DTLZ1a}

The test function DTLZ1a is a two objective test function and has six variables [19]. It is given by:

$$
\begin{aligned}
& \text { minimize } f_{1}=\frac{1}{2} x_{1}(1+g), \\
& \text { minimize } f_{2}=\frac{1}{2}\left(1-x_{1}\right)(1+g), \\
& g=100\left[5+\sum_{i=2}^{6}\left(\left(x_{i}-0.5\right)^{2}-\cos \left(2 \pi\left(x_{i}-0.5\right)\right)\right)\right], \\
& x_{i} \in[0,1], \quad i=1, \ldots, n, \quad n=6 .
\end{aligned}
$$

The Pareto set for this function consists of all solutions where all by the first decision variables are equal to 0.5 and the first decision variable may take any value between 0 and 1 .

For this test function, the value of $\alpha$ for the spreading phase is set to 0.01 . The convergence to the Pareto frontier and the distribution of solutions obtained by LGP, ParEGO and NSGA II for the test function DTLZ1a is depicted in Fig. 17. Different sizes of the objective space are illustrated in order to incorporate all solutions obtained by all techniques. It is obvious that LGP assure a very good convergence and distribution for this function. The convergence of the merit function during the 20 re-starts is depicted in Fig. 18. From the results presented in Table 7 it can be observed that none of the solutions obtained by LGP are dominated neither by ParEGO or by NSGA II, while solutions obtained by LGP dominate all 100 solutions obtained by ParEGO and NSGA II. Ninety-one of the solutions obtained by NSGA II are dominated by solutions obtained by ParEGO while 75 of the solutions obtained by ParEGO are dominated by solutions obtained by NSGA II.

\subsection{Test function DTLZ4a}

Test function DTLZ4a has three-objective functions and eight decision variables and is given by:

$$
\begin{aligned}
& \text { minimize } f_{1}=(1+g) \cos \left(\frac{x_{1}^{100} \pi}{2}\right) \cos \left(\frac{x_{2}^{100} \pi}{2}\right), \\
& \text { minimize } f_{2}=(1+g) \cos \left(\frac{x_{1}^{100} \pi}{2}\right) \sin \left(\frac{x_{2}^{100} \pi}{2}\right), \\
& \text { minimize } f_{3}=(1+g) \sin \left(\frac{x_{1}^{100} \pi}{2}\right), \\
& g=\sum_{i=3}^{8}\left(x_{i}-0.5\right)^{2}, \\
& x_{i} \in[0,1], \quad i=1, \ldots, n, \quad n=8 .
\end{aligned}
$$



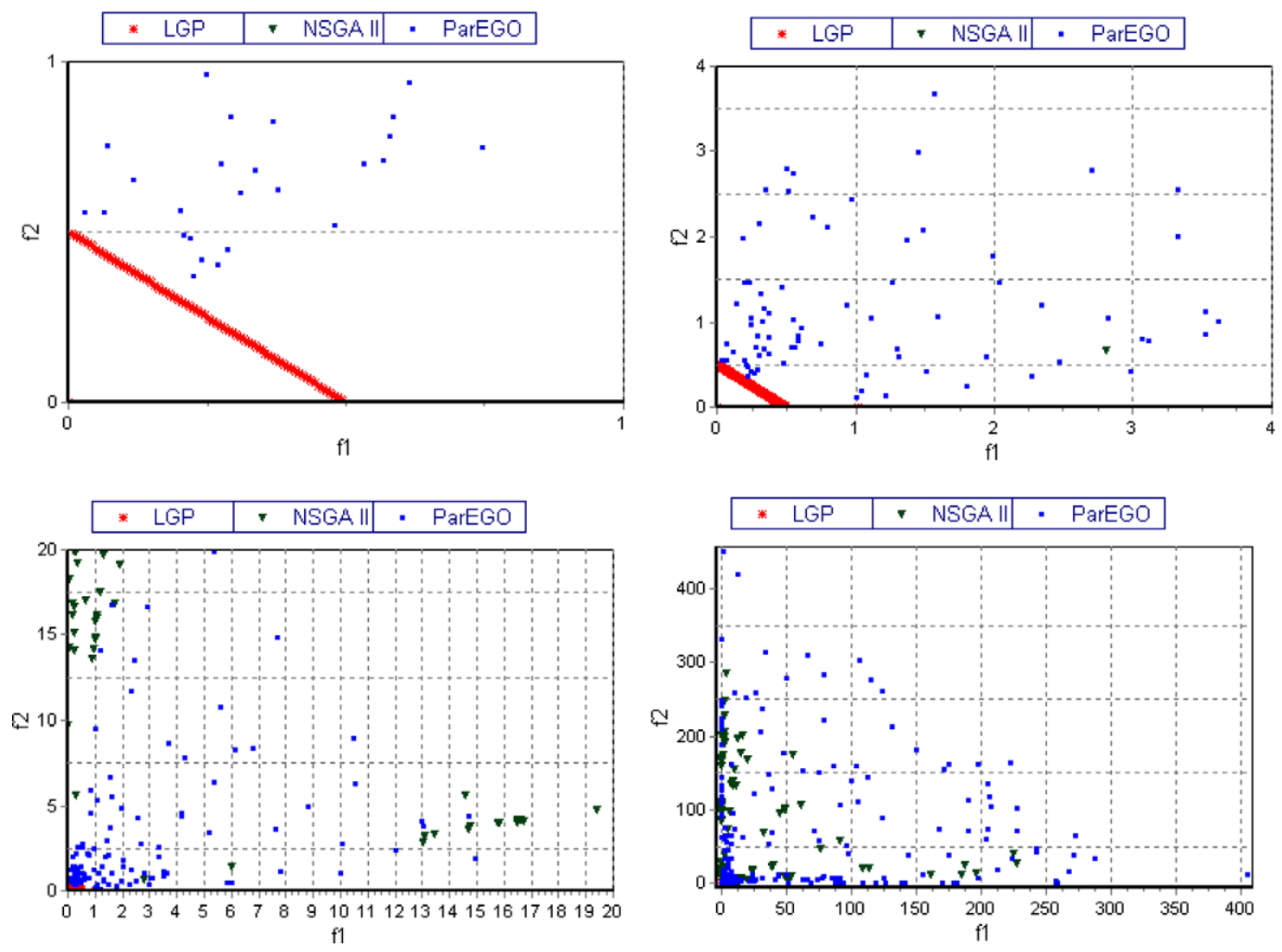

Fig. 17. Distribution of solutions on the Pareto frontier obtained by LGP, ParEGO and NSGA II for test function DTLZ1.

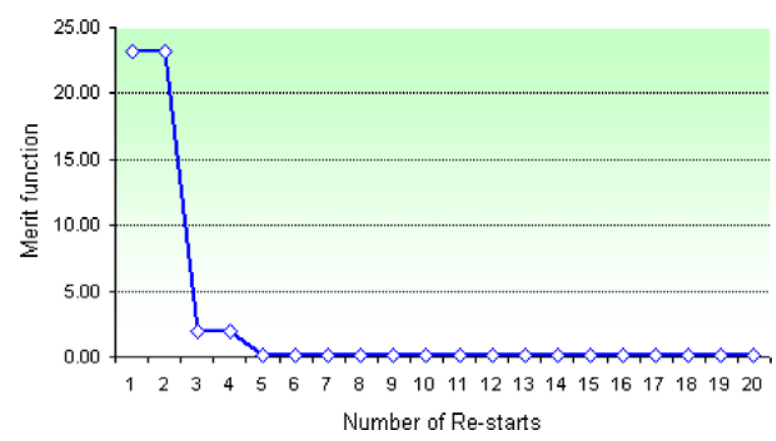

Fig. 18. Behavior of merit function for test function DTLZ1a during the convergence phase.

Table 7

The dominance between solutions obtained by LGP, ParEGO and NSGA II for test function DTLZ1a.

\begin{tabular}{|c|c|c|c|c|c|c|c|c|}
\hline Dominate & ParEGO & NSGA II & Dominate & LGP & NSGA II & Dominate & LGP & ParEGO \\
\hline LGP & 100 & 100 & ParEGO & 0 & 75 & NSGA II & 0 & 91 \\
\hline Dominated & ParEGO & NSGA II & Dominated & LGP & NSGA II & Dominated & LGP & ParEGO \\
\hline LGP & 0 & 0 & ParEGO & 100 & 91 & NSGA II & 100 & 75 \\
\hline
\end{tabular}

The Pareto front is $1 / 8$ of the unit sphere centered in origin. The Pareto optimal set consist of all solutions but the first two decision variables are equal to 0.5 and the first two decision variables may take any value between 0 and 1 .

For test function DTLZ4a the value of $\alpha$ is set to 0.2 . The distribution of solutions on the Pareto frontier and the convergence to the Pareto frontier for all the three algorithms is depicted in Fig. 19. The distribution on the Pareto frontier obtained 

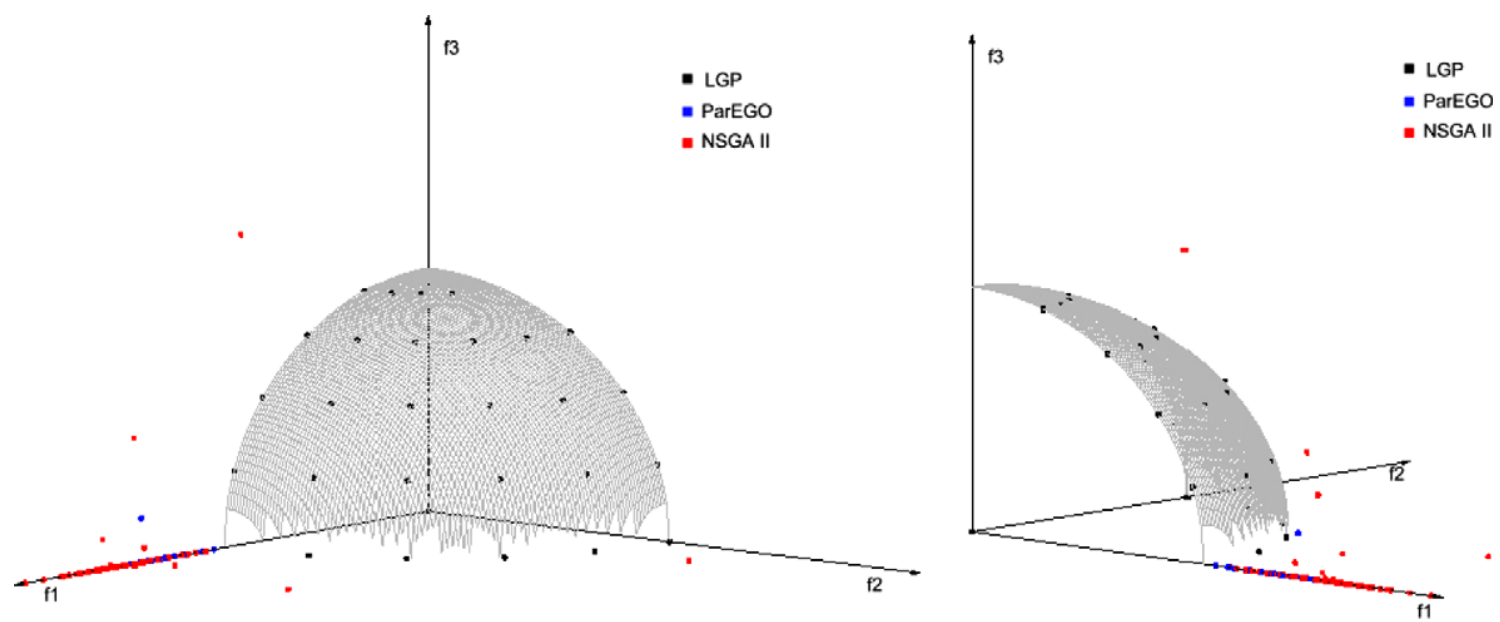

Fig. 19. Convergence to the Pareto frontier and distribution of solutions obtained by LGP, ParEGO and NSGA II on the Pareto frontier for test function DTLZ4a (view from different angles).

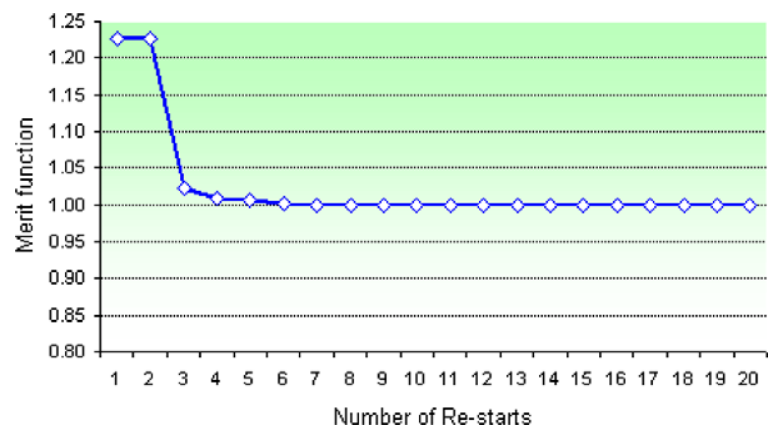

Fig. 20. Behavior of merit function for test function DTLZ4 during the convergence phase.

for different other values of $\alpha$ is depicted in Fig. 21. With a higher number of nondominated solutions (1000) LGP assure a better coverage of the Pareto frontier (as evident from Fig. 22). The convergence of the merit function is depicted in Fig. 20.

From Fig. 19 it can be observed that, compared to ParEGO and NSGA II, LGP is assuring a very good convergence. The latter two approaches are not converging very well with the parameters used.

As evident from Table 8 none of the solutions obtained by LGP are dominated neither by ParEGO or by NSGA II while solutions obtained by LGP dominate all 100 solutions obtained by ParEGO and NSGA II. Ninety-eight of the solutions obtained by NSGA II are dominated by solutions obtained by ParEGO while 60 of the solutions obtained by ParEGO are dominated by solutions obtained by NSGA II.

\subsection{Test function DTLZ7a}

This test function has three objectives and eight decision variables and it is given by:

$$
\begin{aligned}
& \text { minimize } f_{1}=x_{1}, \\
& \text { minimize } f_{2}=x_{2}, \\
& \text { minimize } f_{3}=(1+g) h, \\
& g=1+\frac{9}{6} \sum_{i=3}^{8} x_{i}, \\
& h=3-\sum_{i=1}^{2}\left[\frac{f_{i}}{1+g}\left(1+\sin \left(3 \pi f_{i}\right)\right)\right], \\
& x_{i} \in[0,1], \quad i=1, \ldots, n, \quad n=8 .
\end{aligned}
$$



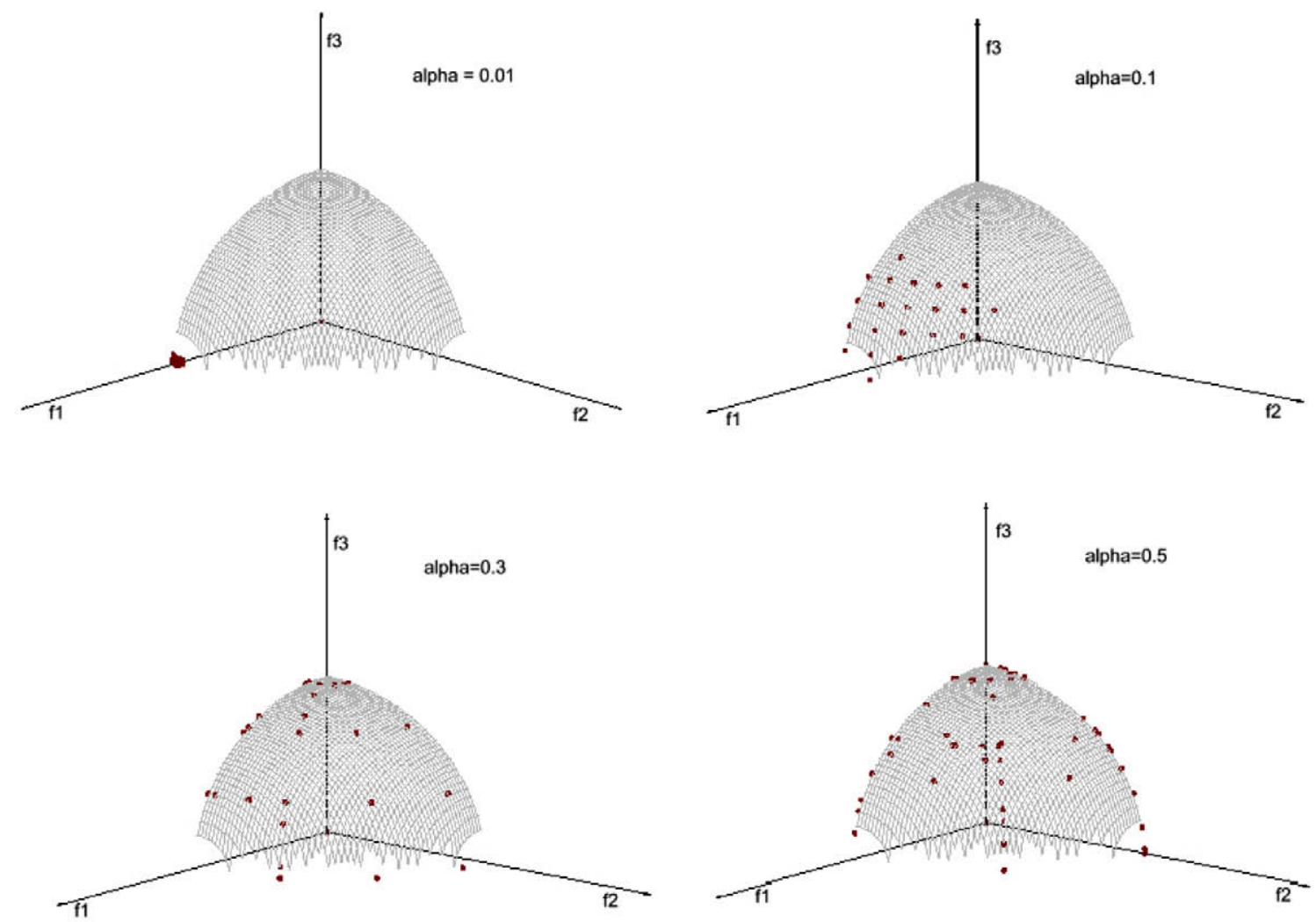

Fig. 21. Distribution of solutions on the Pareto frontier obtained by LGP by considering different values for $\alpha$ in the spreading phase for test function DTLZ4a.

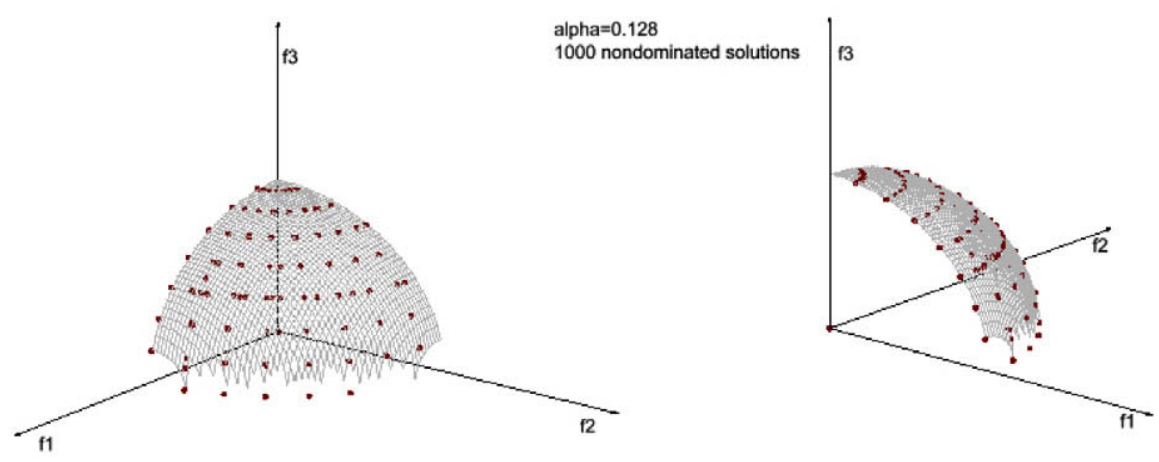

Fig. 22. Distribution of solutions obtained by LGP for DTLZ4 test function considering a set of 1000 nondominated solutions (view from different angles).

The Pareto front has four discontinuous regions and the Pareto set consists of all solutions where all by the first two decision variables are equal to 0 .

The test function DTLZ7a has four discontinuous Pareto regions. LGP is able to converge very well and it is able to spread into the all four disconnected Pareto regions from a single starting point. The value of $\alpha$ used is 1 , but there is not much difference between different values of $\alpha$ as in the case of DTLZ4a test function. Fig. 23 depicts the distribution of LGP solutions for two different values of $\alpha$. As evident from Fig. 24, both ParEGO and NSGA II ar far from the Pareto front in terms of convergence. Forty-four of the solutions obtained by LGP dominates solutions obtained by ParEGO while 15 solutions obtained by ParEGO dominates solutions obtained by LGP. Ninety-one solutions obtained by NSGA II are dominated by solutions obtained by LGP and 20 solutions obtained by LGP are dominated by solutions obtained by NSGA II. Thirty-nine solutions obtained by ParEGO are dominated by solutions obtained by NSGA II while 33 of the solutions obtained by NSGA II are 
Table 8

The dominance between solutions obtained by LGP, ParEGO and NSGA II for test function DTLZ4a.

\begin{tabular}{llllllll}
\hline Dominate & ParEGO & NSGA II & Dominate & LGP & NSGA II & Dominate \\
LGP & 100 & 100 & ParEGO & 0 & 98 & LGP \\
Dominated & ParEGO & NSGA II & Dominated & LGP & NSGA II & Dominated \\
LGP & 0 & 0 & ParEGO & 100 & 60 & LGP & NSGA II \\
\hline
\end{tabular}

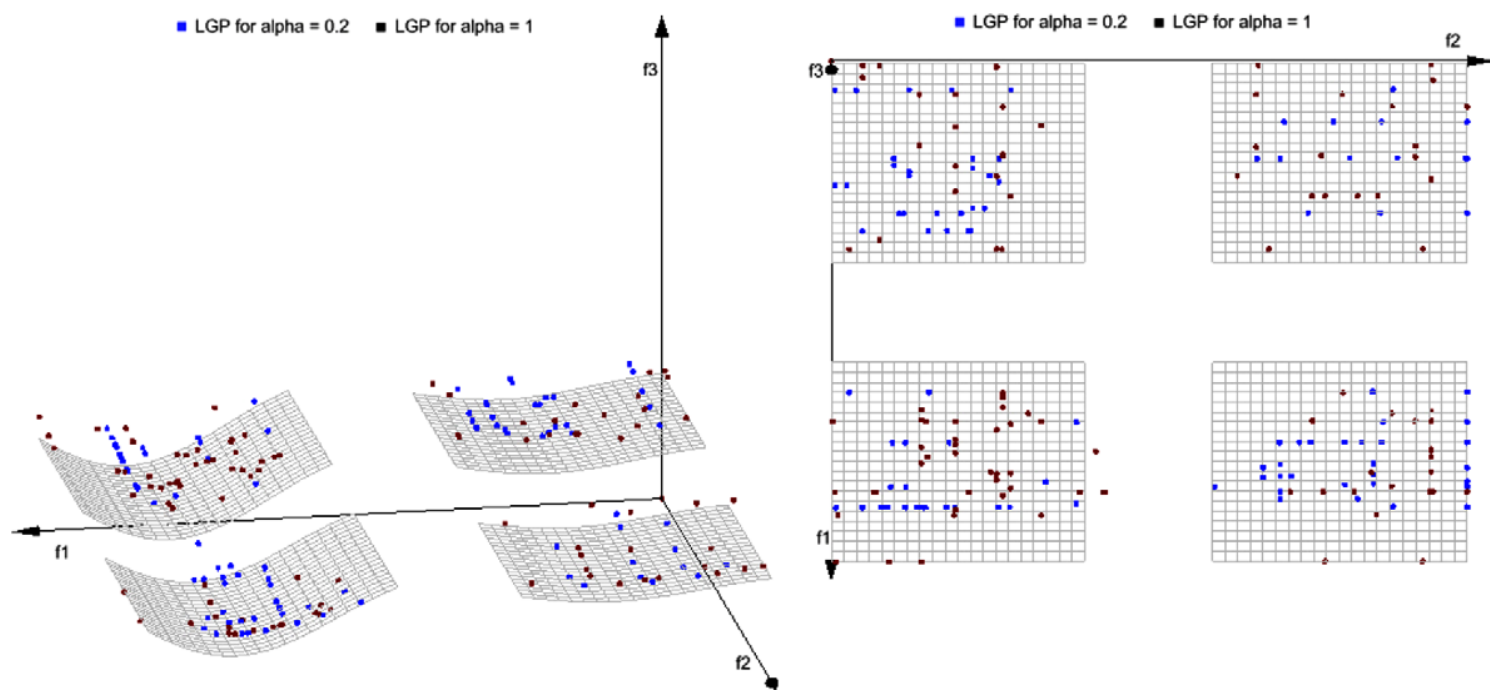

Fig. 23. Distribution of solutions on the Pareto frontier obtained by LGP by considering different values for $\alpha$ in the spreading phase for test function DTLZ7a.

dominated by solutions obtained by ParEGO (as evident from Table 9). The convergence of the merit function is depicted in Fig. 25.

As evident from the graphical representation of the results obtained by all the three techniques, ParEGO and NSGA II and not always providing a very good convergence to the Pareto front. This means that they require a higher number of generations (which, in turn, involves a higher computational time) in order to assure a good convergence to the true Pareto front. LGP converge very fast and require less than $200 \mathrm{~ms}$ (milliseconds) to obtain a set of Pareto solutions. Also, the convergence is very good and not all 20 re-starts are required in the convergence phase (for test functions KNO1 and VLMOP3 4 re-starts are enough while for test functions DTLZ1, DTLZ4 and OKA1 5 re-starts assure the convergence to the first Pareto optimal solution).

\section{Investigation of LGP performances for optimization of a flow injection system for determining hydroquinone}

This problem requires optimizing the determination of hydroquinone by using a flow injection system with amperometric detection. There are three factors that affect the analytical signal:

- the carrier solution flow rate, $\Phi\left(\mathrm{mL} \mathrm{min}^{-1}\right)$;

- the conditioning cell potential, $E_{a}(\mathrm{mV})$;

- the working electrode potential, $E L_{1}(\mathrm{mV})$, of the analytical cell.

The experimental conditions taken are:

- central values equal to $0.75 \mathrm{~mL} / \mathrm{min}$ for the flow rate $\Phi$;

- $-100 \mathrm{mV}$ for the conditioning cell potential, $E_{a}$;

- $175 \mathrm{mV}$ for the working electrode potential, $E L_{1}$, with steps of $0.25 \mathrm{~mL} / \mathrm{min}, 50$ and $125 \mathrm{mV}$.

The task is to find experimental conditions for the three factors so that:

(i) Maximize the signal size estimated as the average value (the peak height of the diagram in $\mu \mathrm{A}$ ) of five signals obtained under the same experimental conditions.

(ii) Minimize the relative variability estimated as the coefficient of variation of the signal, measured as a percentage. 

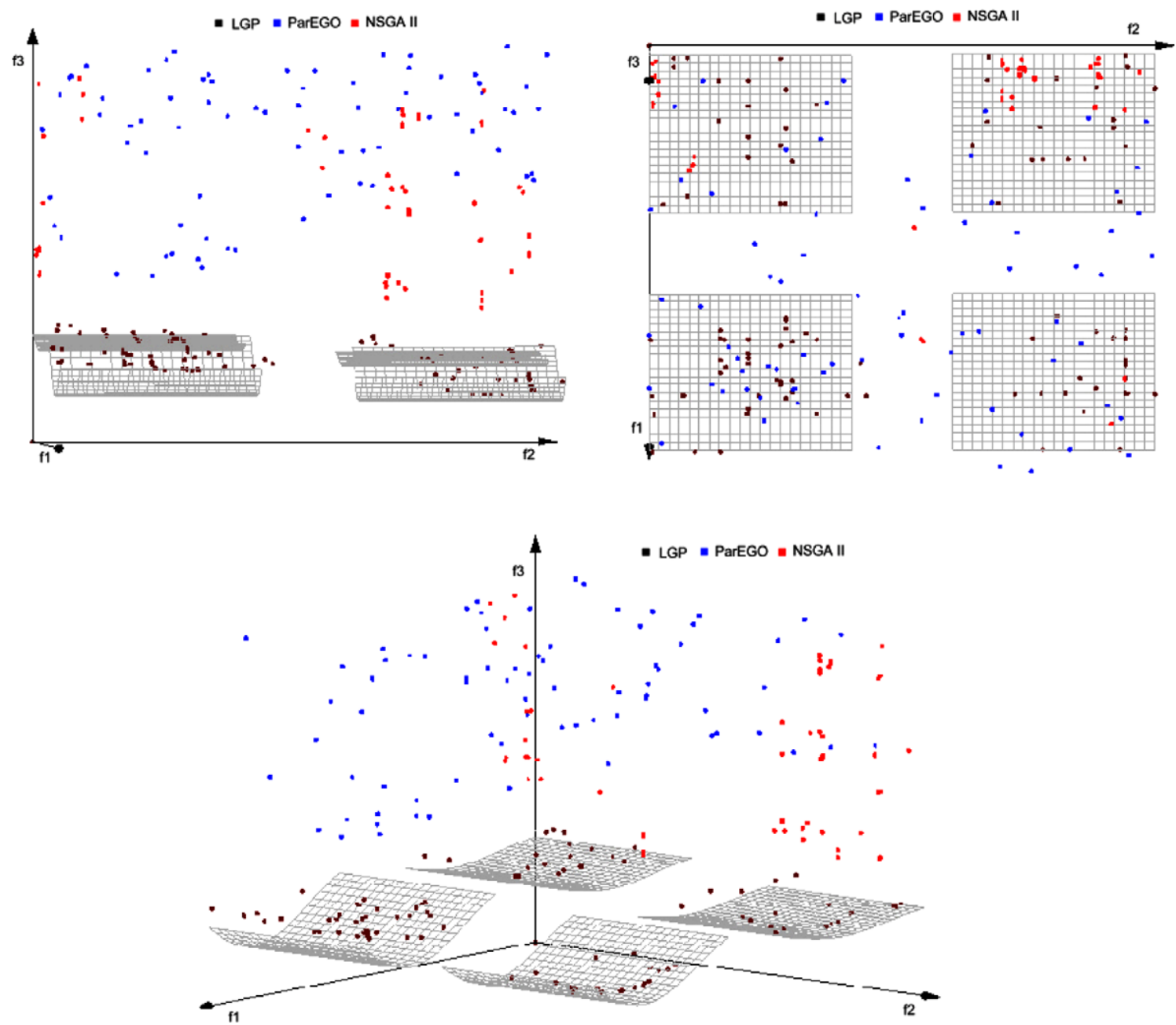

Fig. 24. Convergence to the Pareto frontier and distribution of solutions obtained by LGP, ParEGO and NSGA II on the Pareto frontier for test function DTLZ7a (view from different angles).

Table 9

The dominance between solutions obtained by LGP, ParEGO and NSGA II for test function DTLZ7a.

\begin{tabular}{llllllll}
\hline $\begin{array}{llll}\text { Dominate } \\
\text { LGP }\end{array}$ & ParEGO & NSGA II & Dominate & LGP & NSGA II & Dominate \\
Dominated & 100 & 100 & ParEGO & 0 & LGP & NSGA II \\
LGP & ParEGO & NSGA II & Dominated & LGP & NSGA II & Dominated & LGP \\
\hline
\end{tabular}

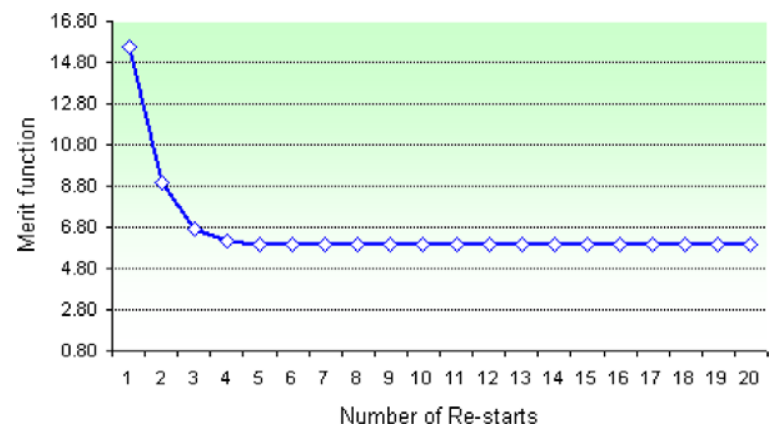

Fig. 25. Behavior of merit function for test function DTLZ7 during the convergence phase. 


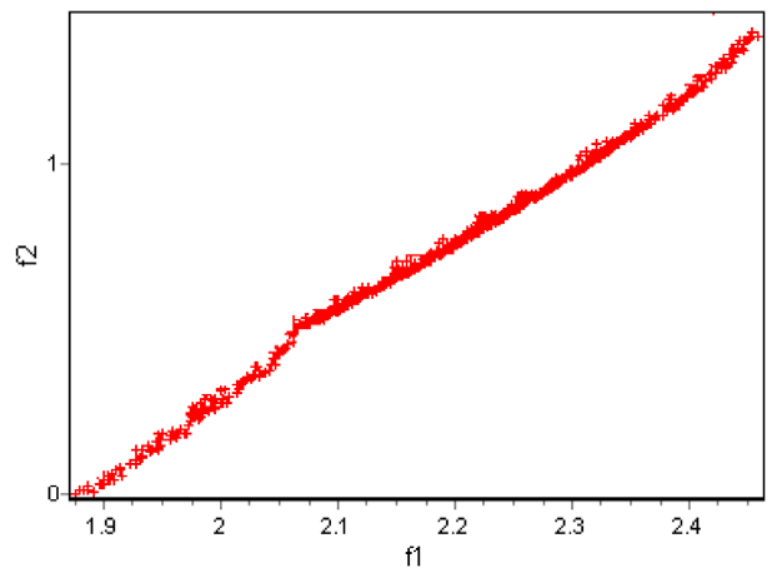

Fig. 26. Pareto-optimal front obtained by LGP for the optimization of a flow injection system for determining hydroquinone.

It is known that, in general, when increasing the size of an analytical signal, its variability is also increased, thus the two objectives are conflicting.

More details about this problem and about the optimization conditions can be found in [32,35]. The authors estimated that the peak height should be below $1.89 \mu \mathrm{A}$ and the coefficient of variation below $1.5 \%$.

By using the notations:

$$
\begin{aligned}
& \Phi \rightarrow x_{1}, \\
& E_{a} \rightarrow x_{2}, \\
& E L_{1} \rightarrow x_{3},
\end{aligned}
$$

the optimization problem can be formulated as follows:

maximize the peak height $(\mu \mathrm{A})$ :

$$
f_{1}=1.51+0.25 x_{1}-0.05 x_{2}+0.06 x_{3}-0.03 x_{1}^{2}+0.02 x_{2}^{2}+0.02 x_{3}^{2}+0.03 x_{1} x_{2}+0.07 x_{1} x_{3}-0.02 x_{2} x_{3} ;
$$

minimize the coefficient of variation (\%):

$$
f_{2}=0.70-0.41 x_{1}-0.13 x_{2}-0.23 x_{3}+0.44 x_{1}^{2}-0.07 x_{2}^{2}+0.13 x_{3}^{2}+0.24 x_{1} x_{2}+0.08 x_{1} x_{3}-0.14 x_{2} x_{3} \text {. }
$$

The mathematical analysis of the models provides the stationary point $(0.49,-0.57,-3.04)$ for the peak height and $(0.55,-0.46,0.47)$ for the coefficient of variation. The stationary point of the model for the peak height is outside the experimental domain, whereas that of the model for the coefficient of variation is inside it. However, both of them are saddle points.

We are applying LGP for solving this optimization problem by considering 10 re-starts and 10 iterations per each re-start.

Fig. 26 shows the Pareto-optimal front (consisting of 1000 solutions) for the peak height (objective $f_{1}$ ) and the coefficient of variation (objective $f_{2}$ ): when the peak height increases, the coefficient of variation also increases and vice versa. The value of $\alpha$ used in the spreading phase is 0.5 .

The corresponding level of factors in the experimental domain is depicted in Fig. 27. In the graphical representation $x_{1}$ corresponds to the carrier solution flow rate $\Phi, x_{2}$ corresponds to the conditioning cell potential $E_{a}$ and $x_{3}$ corresponds to the working electrode potential $E L_{1}$ of the analytical cell.

The results obtained by LGP offer the user the possibility to choose the desired solution based on the expected values for the peak height and the coefficient of variation. Some authors consider that peak heights below $1.5 \mu \mathrm{A}$ are unacceptable and values above $1.8 \mu \mathrm{A}$ are good. Also, they look for values for coefficient of variation below 1.5\% [32]. Desirability values are defined to increase (or decrease) linearly between the two limits. Given all the required conditions for solving this problem, we generated an approximation of the Pareto front and set so that the user can select depending on further preferences.

\section{LGP for the optimization of the formulation of an oral solution}

The application studied in this section comes from pharmaceutics and is described in detail in [20,32]. The problem refers to the formulation of a solution of slightly soluble drug mainly depending on the percentage of surfactant (polysorbate 80), propylene glycol $(\%)$ and invert sugar medium $(\mathrm{mL})$. The central values of the factors that define the experimental domain are $4.0 \%$ of polysorbate $80,20 \%$ of propylene glycol and $55 \mathrm{~mL}$ of sucrose invert medium with steps $0.3 \%$, $3 \%$ and $6 \mathrm{~mL}$, respectively. With a central composite design (spherical and with $\alpha=1.68$ ), two response surfaces (quadratic models) are fitted for the turbidity (ppm) and cloud point $\left({ }^{\circ} \mathrm{C}\right)$ of the resulting solutions. 


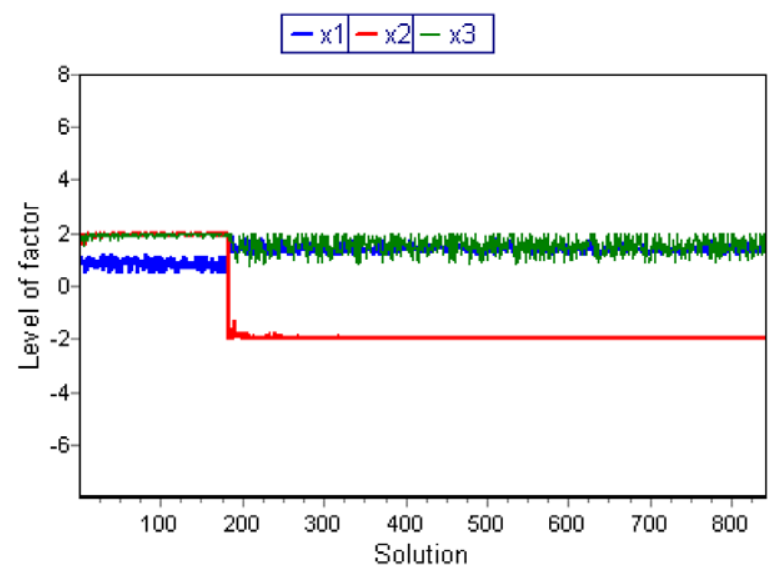

Fig. 27. The level of factors obtained by LGP: $x_{1}$ corresponds to $\Phi, x_{2}$ corresponds to $E_{a}$ and $x_{3}$ corresponds to $E L_{1}$.

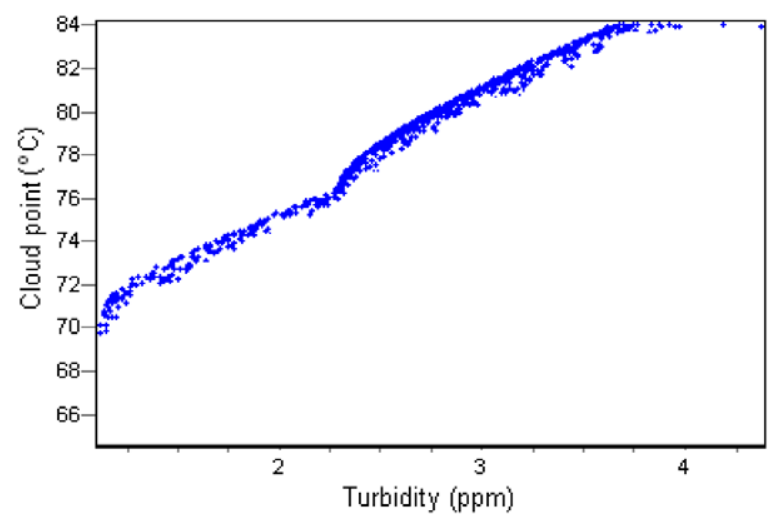

Fig. 28. Pareto front obtained by LGP for turbidity and cloud point conditions.

For fitting the model for the cloud point two experimental points had to be removed with abnormal residuals that produce the regression model to be nonsignificant. These points were, in codified variables $(0,-1.68,0)$ and $(0,1.68,0)$.

Based on some experimental results, the authors of [32] reduced the size of variation of the second factor (propylene glycol) to $[-1.3,1.3]$.

The goal of the fitting of the response surfaces is to find experimental conditions to reduce the turbidity and to increase the cloud point.

By using the notations:

polysorbate $80 \rightarrow x_{1}$,

propylene glycol $\rightarrow x_{2}$,

invert sugar medium $\rightarrow x_{3}$,

the optimization problem can be formulated as follows:

maximize the turbidity:

$$
f_{1}=3.04-0.59 x_{1}-0.15 x_{2}+0.12 x_{3}+0.24 x_{1}^{2}+0.49 x_{2}^{2}-0.06 x_{3}^{2}+0.05 x_{1} x_{2}-0.40 x_{1} x_{3}-0.65 x_{2} x_{3} ;
$$

minimize the cloud point:

$$
f_{2}=72.22+0.84 x_{1}+3.03 x_{2}-5.58 x_{3}+0.63 x_{1}^{2}+1.03 x_{2}^{2}-0.68 x_{3}^{2}-0.15 x_{1} x_{2}+0.92 x_{1} x_{3}-0.72 x_{2} x_{3} .
$$

The analysis of the quadratic models (the standard mathematical methodology) provides the stationary or critical points (points where gradient vector is equal to the null vector) which are $(0.82,-0.23,-0.51)$ for turbidity and $(0.81,-2.24,-2.37)$ for the cloud point. The stationary point for turbidity is inside the experimental domain and that of the cloud point is outside it. In any case, both of them are saddle points (nor maximum nor minimum). As mentioned in [32], according to the generalization (Theorem 2, p. 16 in [2]) of the Weierstrass Extreme Value Theorem (e.g. Theorem 3.9, p. 57 in [33]), both continuous functions in the experimental domain (which is a compact set) attain its maximum and 


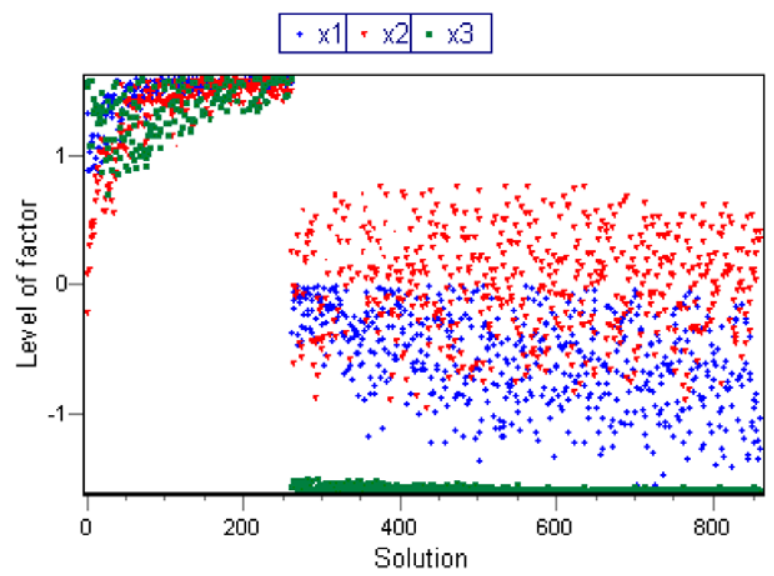

Fig. 29. Solutions (in the variable space) obtained by LGP for the optimization of an oral solution application.

minimum values at points within the compact region; therefore in this case in the boundary of the experimental domain. This theorem, although guaranteeing that there are extreme points in the experimental domain for both models, does not provide any indication about the experimental conditions (the level of the factors) to obtain such extreme points, that means that we do not have any idea about how to move the experimental conditions for minimizing turbidity and maximizing cloud point [32].

From the mathematical experiments performed in [20,32] it is obvious that there are no experimental conditions for the three factors that simultaneously provide the minimum value for turbidity and the maximum value for cloud point. The pairs of values which can be obtained is in the Pareto-optimal front estimated for these two functions depicted in Fig. 24 (the value of $\alpha$ in the spreading phase was set to 1 ).

In the Pareto-optimal front, turbidity ranges from 1.11 to $4.38 \mathrm{ppm}$ and the cloud point from 64.7 to $83.8{ }^{\circ} \mathrm{C}$, but it is clearly observed in Fig. 28 how an increase in cloud point is linked to an increase in turbidity and if we want to decrease turbidity, cloud point is also decreased.

The solutions obtained by LGP are depicted in Fig. 29. Knowing the experimental conditions (inside the experimental domain) that estimate values optimal in one or the other response, the user can choose according to the needs (for instance, solutions for which the turbidity is above some limits and the cloud point is below some limits and vice versa).

\section{Conclusions}

The paper proposed a new approach for multiobjective optimization, which uses an aggregation of objectives and transforms the MOP into a SOP. A line search based technique is applied in order to obtain one solution. Starting from this solution a simplified version of the initial line search is used in order to generate solutions with a well distribution on the Pareto frontier. Numerical experiments performed show that the proposed approach is able to converge very fast and provide a very good distribution (even for discontinuous Pareto frontier) while compared with state of the art population-based metaheuristics such as ParEGO and NSGA II.

Compared to NSGA II and ParEGO, LGP has only few parameters to adjust. It is computationally inexpensive, taking less than $200 \mathrm{~ms}$ to generate a set of nondominated solutions well distributed on the Pareto frontier.

We also considered two practical problems to illustrate the performance of LGP. The first problem refers to the optimization of a flow injection system for determining hydroquinone. LGP is able to generate a very good approximation of the Pareto front and provide results which clearly fulfill all the problem requirements. The second application is from the pharmaceutical design and requires the optimization of an oral solution. LGP obtained a good Pareto front and thus offering the user the possibility to select from a wide range of solutions.

The only inconvenience is that LGP involves first partial derivatives, which make it be restricted to a class of problems which are continuous twice differentiable. One of the further work ideas is to extend LGP to deal with constraint multiobjective optimization problems.

\section{Acknowledgement}

The first author acknowledges the support from the research grant CNCSIS IDEI 2412, Romania.

\section{References}

[1] A. Abraham, L. Jain, R. Goldberg (Eds.), Evolutionary Multiobjective Optimization: Theoretical Advances and Applications, 12 Chapters, Springer Verlag, London, 2005, pp. 135, ISBN 1852337877, 173 Illustrations. 
[2] V. Batanović, D. Petrović, R. Petrović, Fuzzy logic based algorithms for maximum covering location problems, Information Sciences 179 (1-2) (2009) $120-129$.

[3] W. Chen, M.M. Wiecek, J. Zhang, Quality utility - a compromise programming approach to robust design, Journal of Mechanical Design 121 (1999) $179-187$.

[4] I. Das, J. Dennis, A closer look at drawbacks of minimizing weighted sums of objective for Pareto set generation in multicriteria optimization problems, Structural Optimization 14 (1997) 63-69.

[5] I. Das, An improved technique for choosing parameters for Pareto surface generation using normal-boundary intersection, in: Short Paper Proceedings of the Third World Congress of Structural and Multidisciplinary Optimization, vol. 2, 1999, pp. 411-413.

[6] I. Das, J.E. Dennis, Normal Boundary Intersection: a new method for generating the Pareto surface in multicriteria optimization problems, SIAM Journal of Optimization 8 (3) (1998) 631-657.

[7] I. Das, On characterizing the 'knee' of the Pareto curve based on Normal-Boundary Intersection, Structural Optimization 18 (2/3) (1999) 107-115.

[8] K. Deb, A. Pratap, S. Agarwal, T. Meyarivan, A fast and elitist multi-objective genetic algorithm: NSGA-II, IEEE Transaction on Evolutionary Computation 6 (2) (2002) 181-197.

[9] K. Deb, L. Thiele, M. Laumanns, E. Zitzler, Scalable multi-objective optimization test problems, in: Proceedings of the Congress on Evolutionary Computation (CEC-2002), Honolulu, USA, 2002, pp. 825-830.

[10] K. Deb, Multiobjective Optimization Using Evolutionary Algorithms, Series in Systems and Optimization, John Wiley and Sons, Chichester, England, 2001.

[11] M. Ehrgott, X. Gandibleux, Multiobjective Combinatorial Optimization, Multiple-Criteria Optimization: State of the Art Annotated Bibliographic Surveys, Kluwer Academic Publishers, Boston, MA, 2002.

[12] M. Ehrgott, M.M. Wiecek, Mutiobjective programming, in: J. Figueira et al. (Eds.), Multiple Criteria Decision Analysis: State of the Art Surveys, International Series in Operations Research \& Management Science, vol. 78, Springer, New York, 2005.

[13] N. Gould, An introduction to algorithms for continuous optimization, Oxford University Computing Laboratory Notes, 2006.

[14] C. Grosan, A. Abraham, A novel global optimization technique for high dimensional functions, International Journal of Intelligent Systems 24 (4) (2009) $421-440$.

[15] C. Grosan, A. Abraham, Hybrid line search for multiobjective optimization, in: R. Perrott et al. (Eds.), International Conference on High Performance Computing and Communications (HPCC-07), LNCS 4782, Houston, USA, Springer Verlag, Germany, 2007, pp. 62-73.

[16] P.Y. Ho, K. Shimizu, Evolutionary constrained optimization using an addition of ranking method and a percentage-based tolerance value adjustment scheme, Information Sciences 177 (14-15) (2007) 2985-3004.

[17] S. Huband, P. Hingston, L. Barone, L. While, A review of multiobjective test problems and a scalable test problem toolkit, IEEE Transactions on Evolutionary Computation 10 (5) (2006) 477-506.

[18] J. Jahn, Vector Optimization: Theory, Applications, and Extensions, Springer-Verlag, Berlin, 2004.

[19] J. Knowles, L. Thiele, E. Zitzler, A tutorial on the performance assessment of stochastic multiobjective optimizers, TIK Report No. 214, Institute fur Technische Informatik und Kommunikationsnetze ETH Zurich, 2005.

[20] G. Lewis, D. Mathieu, R. Phan-Tan-Luu, Pharmaceutical Experimental Design, Marcel Decker, New York, 2000.

[21] A. Messac, C.A. Mattson, Generating well-distributed sets of Pareto points for engineering design using physical programming, Optimization and Engineering 3 (2002) 431-450.

[22] A. Messac, Physical programming: effective optimization for computational design, AIAA Journal 34 (1) (1996) 149-158.

[23] A. Messac, E. Melachrinoudis, C.P. Sukam, Mathematical and pragmatic perspectives of physical programming, AIAA Journal 39 (5) (2001) 885-893.

[24] A. Messac, C.A. Mattson, Generating well-distributed sets of Pareto points for engineering design using physical programming, Optimization and Engineering 3 (4) (2002) 431-450.

[25] A. Messac, A. Ismail-Yahaya, Multiobjective robust design using physical programming, structural and multidisciplinary optimization, Journal of the International Society of Structural and Multidisciplinary Optimization (ISSMO) 23 (5) (2002) 357-371.

[26] A. Messac, W.M. Batayneh, A. Ismail-Yahaya, Production planning optimization with physical programming, Engineering Optimization 34 (4) (2002) $323-340$

[27] A. Messac, M. Martinez, T. Simpson, Effective product family design using physical programming, Engineering Optimization 34 (3) (2002) $245-261$.

[28] A. Messac, A. Ismail-Yahaya, C.A. Mattson, The normalized normal constraint method for generating the Pareto frontier, Structural and Multidisciplinary Optimization 25 (2) (2003) 86-98.

[29] A. Messac, C.A. Mattson, Normal constraint method with guarantee of even representation of complete Pareto frontier, AIAA Journal 42 (10) (2004) 2101-2111.

[30] K.M. Miettinen, Nonlinear Multiobjective Optimization, Kluwer Academic Publishers, Norwell, 1999.

[31] T. Okabe, Y. Jin, M. Olhofer, B. Sendhoff, On test functions for evolutionary multiobjective optimization, in: Proceedings of Eighth Conference on Parallel Problems Solving from Nature, Springer-Verlag, Germany, 2004, pp. 792-802.

[32] M.C. Ortiz, L. Sarabia, A. Herrero, M.S. Sánchez, Vectorial optimization as a methodological alternative to desirability function, Chemometrics and Intelligent Laboratory Systems 83 (2006) 157-168.

[33] M.H. Protter, Basic Elements of Real Analysis, Undergraduate Texts in Mathematics, Springer-Verlag, New York Inc., 1998.

[34] M.G.C. Resende, J.P. de Sousa (Eds.), Metaheuristics: Computer Decision-Making, Kluwer Academic Publishers, The Netherlands, 2004

[35] M.E. Rueda, L.A. Sarabia, A. Herrero, M.C. Ortiz, Optimisation of a flow injection system with electrochemical detection using the desirability function: application to the determination of hydroquinone in cosmetics, Analytica Chimica Acta 479 (2003) $173-184$.

[36] S. Ruzika, M.M. Wiecek, Approximation methods in multiobjective programming, Journal of Optimization Theory and Applications 126 (3) (2005) 473501.

[37] J. Sanchis, M.A. Martínez, X. Blasco, Integrated multiobjective optimization and a priori preferences using genetic algorithms, Information Sciences 178 (4) (2008) 931-951.

[38] S. Sayın, Measuring the quality of discrete representations of efficient sets in multiple objective mathematical programming, Mathematical Programming Series A 87 (2000) 543-560.

[39] P.K. Tripathi, S. Bandyopadhyay, S.K. Pal, Multi-Objective Particle Swarm Optimization with time variant inertia and acceleration coefficients, Information Sciences 177 (22) (2007) 5033-5049.

[40] D.A.V. Veldhuizen, G.B. Lamont, Multiobjective evolutionary algorithm test suites, in: J. Caroll et al. (Eds.), Proceedings of ACM Symposium on Applied Computing, San Antonio, Texas, 1999, pp. 351-357.

[41] Y. Wang, Y. Yang, Particle swarm optimization with preference order ranking for multi-objective optimization, Information Sciences 179 (12) (2009) 1944-1959. 